LA-14349

Approved for public release;

distribution is unlimited.

Slant Path Distances Through

Cells in Cylindrical Geometry and an

Application to the Computation of Isophotes

- Los Alamos 
Funding is provided by the Department of Energy, Nonproliferation Office.

Los Alamos National Laboratory, an affirmative action/ equal opportunity employer, is operated by Los Alamos National Security, LLC, for the National Nuclear Security Administration of the U.S. Department of Energy under contract DE-AC52-06NA25396.

\section{MAS NASA \\ National Nuclear Security Administration}

This report was prepared as an account of work sponsored by an agency of the U.S. Government. Neither Los Alamos National Security, LLC, the U.S. Government nor any agency thereof, nor any of their employees make any warranty, express or implied, or assume any legal liability or responsibility for the accuracy, completeness, or usefulness of any information, apparatus, product, or process disclosed, or represent that its use would not infringe privately owned rights. Reference herein to any specific commercial product, process, or service by trade name, trademark, manufacturer, or otherwise does not necessarily constitute or imply its endorsement, recommendation, or favoring by Los Alamos National Security, LLC, the U.S. Government, or any agency thereof. The views and opinions of authors expressed herein do not necessarily state or reflect those of Los Alamos National Security, LLC, the U.S. Government, or any agency thereof. Los Alamos National Laboratory strongly supports academic freedom and a researcher's right to publish; as an institution, however, the Laboratory does not endorse the viewpoint of a publication or guarantee its technical correctness. 
LA-14349

Issued: December 2007

Slant Path Distances Through

Cells in Cylindrical Geometry and an

Application to the Computation of Isophotes

Rodney Whitaker

Eugene Symbalisty

- Los Alamos 



\title{
Slant Path Distances Through Cells in Cylindrical Geometry and an Application to the Computation of Isophotes
}

\author{
by \\ Rodney Whitaker and Eugene Symbalisty
}

an updated version of the original unpublished report by Henry G. Horak and John W. Kodis 


\section{Contents}

1 Introduction $\quad 1$

2 Slant Path Geometry $\quad 1$

2.1 Right Circular Cone . . . . . . . . . . . . . . . . . . . 4

2.2 Right Circular Cylinder . . . . . . . . . . . . . . . . . . 4

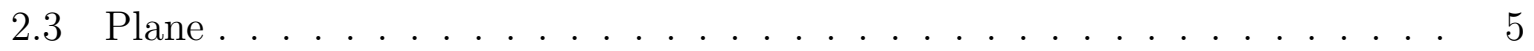

3 The Subroutine HOWFAR $\quad 5$

4 The Subroutine WHERE $\quad 6$

$\begin{array}{lll}5 & \text { The Calculation of Isophotes } & 7\end{array}$

6 Current Work $\quad 10$

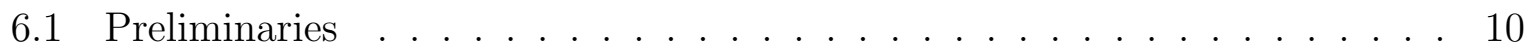

6.2 Isophote Application . . . . . . . . . . . . . . . . . . . 11

6.3 Particular Cases . . . . . . . . . . . . . . . . . . . . . . . . 14

$\begin{array}{lll}7 & \text { Summary } & 19\end{array}$

8 Acknowledgements $\quad 19$

9 Bibliography $\quad 20$

10 Appendix $\quad 21$

10.1 Sample input file . . . . . . . . . . . . . . . . . . . . 21

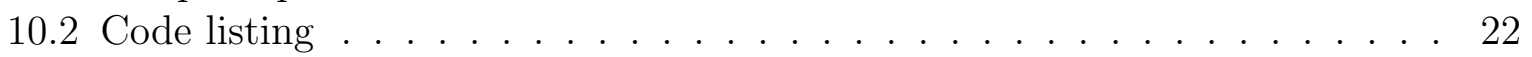

10.3 OpenMP make file on a MAC G5 . . . . . . . . . . . . . . . 50

10.4 Make file for serial processor such as Sun . . . . . . . . . . . . . . 50 


\section{List of Figures}

1 The position vector, a, and the direction vector, $\Omega . \ldots . \ldots 2$

2 A quadrilateral mesh cell. . . . . . . . . . . . . . . . 2

3 The incident, $I_{\nu}(0, \Omega)$, and emergent, $I_{\nu}(s, \Omega)$, intensities. . . . . . . . 7

4 The observer, $\mathrm{O}$, is located at $\left(a_{0}, c_{0}\right) \ldots \ldots \ldots \ldots$

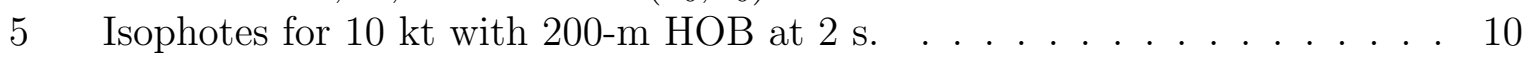

6 Isophotes for a 1-kt burst at 50-m HOB and a time of $0.4 \mathrm{~s}$, horizontal path 12

7 Isophotes for a 1-kt burst at 50-m HOB and a time of $0.4 \mathrm{~s}$, and $45^{0}$ path. 13

8 The Si band power vs time curve for $45^{0}$ look angle. . . . . . . . . . . . . . 14

9 Si flux versus time for the $10 \mathrm{kt}, 240-\mathrm{m}$ HOB at three elevation angles. . . 15

10 Si flux versus time for the $10 \mathrm{kt}, 0-\mathrm{m} \mathrm{HOB}$ at three elevation angles. . . . . 16

11 Isophotes for a $10 \mathrm{kt}$ burst at $0-\mathrm{m}$ HOB and $0^{0}$ elevation angle. . . . . . . 16

12 Isophotes for a 10-kt burst at $0-\mathrm{m}$ HOB and $90^{\circ}$ elevation angle. . . . . . . 17

13 Si flux versus time for the $10 \mathrm{kt}, 0-\mathrm{m}$ and 240-m HOB showing the delayed minimum time for the $0-\mathrm{m}$ HOB case. . . . . . . . . . . . . . . . . 18 


\title{
1 Introduction
}

This report is composed of two parts. The first is a restoration of the hardcopy draft of the original report by Horak and Kodis from 1983 that did not get to final form. The algorithm documented in that draft was in routine use in weapon effects calculations at Los Alamos since the mid-1970s. That draft was converted to LaTex format by Rod Whitaker and Eugene Symbalisty in late 2006, and C. Flaming made electronic versions of the original figures. We give first the text and figures of the original report (Sections 1 through 5) with new results starting after Section 5.

\begin{abstract}
In computer programs involving two-dimensional cylindrical geometry, it is often necessary to calculate the slant path distance in a given direction from a point to the boundary of a mesh cell. A subroutine, HOWFAR, has been written that accomplishes this, and is very economical in computer time. An example of its use is given in constructing the isophotes for a low altitude nuclear fireball.
\end{abstract}

Computer programs that solve problems in two-dimensional cylindrical geometry often must calculate slant path distances through mesh cells (see, e.g., Amsden and Hirt, 1973; Anderson and Sandford, 1974, Horak et al., 1982, and Lathrop and Brinkley, 1973). This is particularly important in Monte Carlo computations that follow the random walks of numerous statistical particles. A subroutine, $H O W F A R$ has been written that efficiently calculates the slant path distance in a given direction from a point to the boundary of a mesh cell, and allows for the many special cases. HOWFAR has become very valuable in several radiation-hydrodynamics codes that are used at Los Alamos to follow the evolution of nuclear explosions in the earth's atmosphere (Anderson and Sandford, 1974, Horak et al., 1982).

In Section $\mathrm{V}$ the use of HOWFAR is illustrated to find slant optical distances through cells, and by using the formal solution of the equation of transfer to calculate isophotes (radiance contours) for a low-altitude nuclear fireball.

\section{Slant Path Geometry}

The basic formulas for calculating slant path distances through mesh cells in cylindrical geometry are readily obtained by using vector algebra. Refer to Figures 1 and 2. The cross section of a cell in the XZ-plane is the area bounded by four straight-line segments; the entire cell is the volume described by rotating this figure in a circle about the OZ-axis. This quadrilateral is assumed to be convex, with the four vertices numbered consecutively $\mathrm{i}=1,2,3,4$ in the counterclockwise sense as viewed from a point on the negative Y-axis. In Lagrangian hydrodynamic calculations the mesh cells can be subject to severe distortion, although provision is usually provided to prevent cell boundaries from becoming concave.

The position vector, a, of a moving point at a given instant of time can be written 


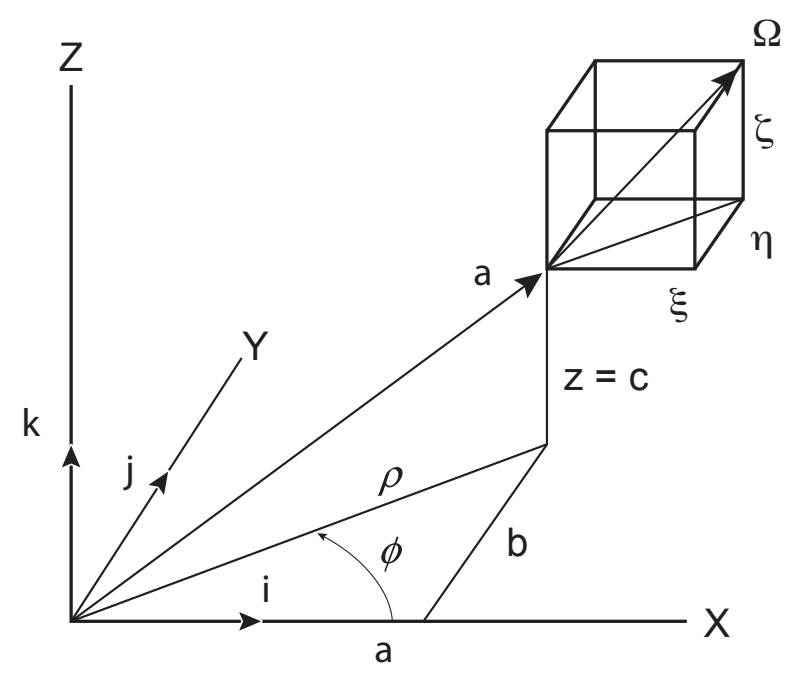

Figure 1: The position vector, a, and the direction vector, $\boldsymbol{\Omega}$.

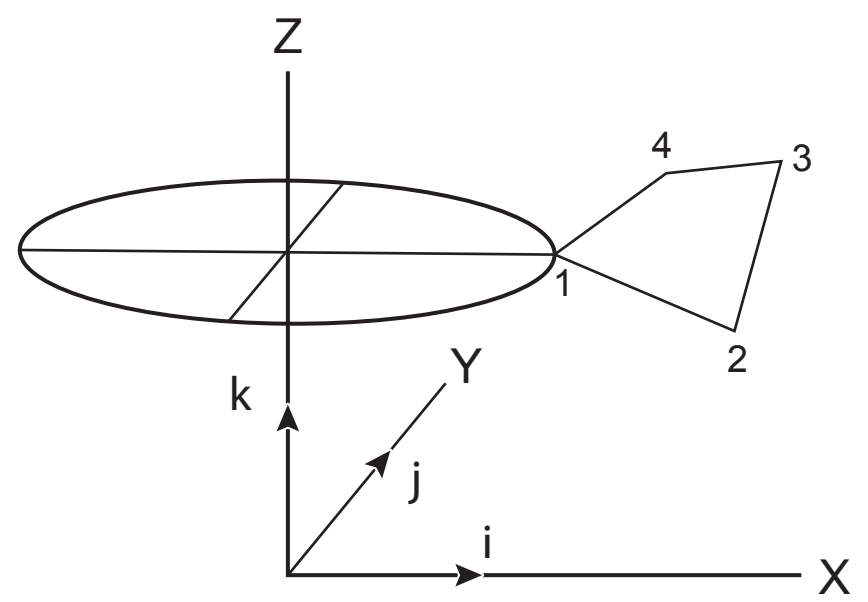

Figure 2: A quadrilateral mesh cell. 


$$
\mathbf{a}=a \mathbf{i}+b \mathbf{j}+c \mathbf{k}
$$

where $\mathbf{i}, \mathbf{j}$, and $\mathbf{k}$ are basic unit vectors in a right-handed orthogonal Cartesian coordinate system, and its direction of motion is given by the unit vector

$$
\boldsymbol{\Omega}=\xi \mathbf{i}+\eta \mathbf{j}+\zeta \mathbf{k} .
$$

The vector equation of the straight line segment originating at the point $\mathbf{a}$, and extending in the direction $\Omega$ is

$$
\mathbf{r}(d)=\mathbf{a}+\Omega d \quad(d>0),
$$

where $\mathbf{r}=x \mathbf{i}+y \mathbf{j}+z \mathbf{k}$ is the position vector of any point on the line segment, and $d$ is the distance parameter. The geometric problem is to find the points of intersection of this line segment with the boundary surfaces of the mesh cells, viz., right circular cones and cylinders described about the OZ-axis, and planes perpendicular to the OZ-axis. It is assumed throughout that the moving point in question is located within a mesh cell, although it may be necessary to perform some computation in order to identify the precise cell (refer to Sec. IV).

Let $(\rho, \phi, z)$ be the cylindrical coordinates of a point $\mathrm{P}$, and $\mathbf{x}_{i}, \mathbf{x}_{i}+\mathbf{d} \mathbf{x}_{i}(\mathrm{i}=1,2,3,4)$ the position vectors in the XZ-plane of two consecutive vertices of a mesh cell, where

$\mathbf{x}_{i}=x_{i} \mathbf{i}+z_{i} \mathbf{k}$, and $\mathbf{d} \mathbf{x}_{i}=d x_{i} \mathbf{i}+d z_{i} \mathbf{k}$. The equation of a right circular cone whose line of symmetry is the Z-axis, and which passes through the points $\mathbf{x}_{i}, \mathbf{x}_{i}+\mathbf{d} \mathbf{x}_{i}$ is

$$
\mathbf{r}(\rho, \phi)=\rho \cos \phi \mathbf{i}+\rho \sin \phi \mathbf{j}+\left(z_{0}+\rho \cot \alpha\right) \mathbf{k},
$$

where

$$
z_{0}=z_{i}-x_{i} \cot \alpha
$$

and

$$
\cot \alpha=\frac{d z_{i}}{d x_{i}}
$$

$\alpha$ is the semivertex angle of the cone, and $z_{0} \mathbf{k}$ the position vector of the vertex. If $d x_{i}=0$, the resulting equations describe a right circular cylinder:

$$
\mathbf{r}(\phi, z)=\rho_{0} \cos \phi \mathbf{i}+\rho_{0} \sin \phi \mathbf{j}+z \mathbf{k}
$$

where

$$
\rho_{0}=x_{i}, \quad d x_{i}=0 .
$$

The equation of a plane perpendicular to the z-axis, and that contains the points $\mathbf{x}_{i}, \mathbf{x}_{i}+d x_{i} \mathbf{i}$, is 


$$
\mathbf{r}(\rho, \phi) \cdot \mathbf{k}=z_{0}
$$

where

$$
z_{0}=z_{i}, \quad d z_{i}=0 .
$$

The points of intersection e of the above surfaces with the straight line segment $\mathbf{r}=$ $\mathbf{a}+\Omega d$ are found by simultaneous solution, and the results are described below.

\subsection{Right Circular Cone}

For a right circular cone, we have

$$
\begin{aligned}
\mathbf{e} & =e \mathbf{i}+f \mathbf{j}+g \mathbf{k} \\
& =(a+\xi d) \mathbf{i}+(b+\eta d) \mathbf{j}+(c+\zeta d) \mathbf{k}
\end{aligned}
$$

where

$$
\begin{aligned}
d & =\frac{-Q \pm \sqrt{\left(Q^{2}-P R\right)}}{P} \\
P & =\xi^{2}+\eta^{2}-F^{2} \\
Q & =a \xi+b \eta-E F \\
R & =a^{2}+b^{2}-E^{2} \\
E & =\left(c-z_{0}\right) \tan \alpha \\
F & =\xi \tan \alpha \\
z_{o} & =z_{i}-x_{i} \cot \alpha \\
\cot \alpha & =\frac{d z_{i}}{d x_{i}} .
\end{aligned}
$$

\subsection{Right Circular Cylinder}

For the case of the right circular cylinder, the above equations for e and $d$ apply with

$$
\begin{aligned}
P & =\xi^{2}+\eta^{2} \\
Q & =a \xi+b \eta \\
R & =a^{2}+b^{2}-x_{i}^{2} .
\end{aligned}
$$




\subsection{Plane}

For the case of the plane, equation 11 applies with

$$
d=\frac{z_{i}-c}{\zeta}
$$

There are special cases that can arise and must be properly treated in the program; some of these will be discussed subsequently.

\section{The Subroutine HOWFAR}

A FORTRAN listing of the subroutine HOWFAR is given in the appendix. It will be necessary for the user to provide statements regarding the memory storage and location of coordinates, etc., and to set up the appropriate common blocks. Comments have been liberally inserted, but certain aspects require more discussion.

A test is made at the outset whether the point $\mathrm{P}$ with position vector a really lies inside the cell in which it is surmised to be located. Again let $\mathbf{x}_{\mathbf{i}}=x_{i} \mathbf{i}+z_{i} \mathbf{k}$, and $\mathbf{x}_{\mathbf{i}}+\mathbf{d} \mathbf{x}_{\mathbf{i}}$, where $\mathbf{d} \mathbf{x}_{\mathbf{i}}=d x_{i} \mathbf{i}+d z_{i} \mathbf{k}$, be the position vectors of any two consecutive mesh cell vertices in the XZ-plane. The plane POZ can be rotated about axis OZ into coincidence with the XOZ reference plane; thus $P$ maps onto $P^{\prime}$ so that the position vector of $P^{\prime}$ is $\mathbf{a}^{\prime}=\rho \mathbf{i}+c \mathbf{k}$, where $\rho=\sqrt{a^{2}+b^{2}}$. The scalar triple product

$$
C=\left[\left(\mathbf{a}^{\prime}-\mathbf{x}_{\mathbf{i}}\right) \times \mathbf{d} \mathbf{x}_{\mathbf{i}}\right] \cdot \mathbf{j},
$$

serves to indicate in which half of the XZ-plane, as divided by the line $\mathbf{x}_{\mathbf{i}}+t \mathbf{d} \mathbf{x}_{\mathbf{i}}$ ( $\mathrm{t}$ is a variable scalar), the point $P^{\prime}$ is to be found. If $C$ is positive, $P^{\prime}$ is said to lie in the left half plane. If $P^{\prime}$ is to the left of all four cell sides $\left(\mathbf{d} \mathbf{x}_{\mathbf{i}}=\mathbf{x}_{\mathbf{2}}-\mathbf{x}_{\mathbf{1}}, \mathbf{x}_{\mathbf{3}}-\mathbf{x}_{\mathbf{2}}, \mathbf{x}_{\mathbf{4}}-\mathbf{x}_{\mathbf{3}}, \mathbf{x}_{\mathbf{1}}-\mathbf{x}_{\mathbf{4}}\right.$ respectively), it is clearly inside the cell. If $C$ is not positive for any one of the cell sides, then $P^{\prime}$, and therefore $P$, must lie outside the cell. In this latter case, the subroutine WHERE (see the next section) is called to identify the proper cell.

In order to find the distance from $P(\mathbf{a})$ in the direction $\Omega$ to the emergence point, it is necessary to find the minimum positive distance among the intersections with the four cell surfaces. In doing this, whenever imaginary values of $D$ occur, they need only be identified, and not calculated. The choice between two positive real roots is complicated because of a possible intersection with the false cone. The latter is that half of the cone not containing the two given mesh points, but nevertheless defined by the same second degree equation. There is a simple procedure that can be used in computations to separate true and false cone solutions. An intersection point e of the straight line and cone, obtained from equations 11 and 13 , is given by $\mathbf{e}=\mathbf{a}+\boldsymbol{\Omega} d=e \mathbf{i}+f \mathbf{j}+g \mathbf{k}=\rho \mathbf{i}^{\prime}+g \mathbf{k}$, where $\rho \mathbf{i}^{\prime}=e \mathbf{i}+f \mathbf{j}$ and $\rho=\sqrt{e^{2}+f^{2}}$. Also, the cone line-element through e passes through the point $\mathbf{P}_{\mathbf{i}}=x_{i} \mathbf{i}^{\prime}+z_{i} \mathbf{k}$ in the direction $\mathbf{d p}_{\mathbf{i}}=d x_{i} \mathbf{i}^{\prime}+d z_{i} \mathbf{k}$. The condition that $\mathbf{e}$ lies on this line-element is $\left(\mathbf{e}-\mathbf{p}_{\mathbf{i}}\right) \times \mathbf{d p}_{\mathbf{i}} \cdot \mathbf{j}^{\prime}=0$ with $\mathbf{j}^{\prime}=\mathbf{k} \times \mathbf{i}^{\prime}$, or simply

$$
\left(\rho-x_{i}\right)-\left(g-z_{i}\right)\left(\frac{d x_{i}}{d z_{i}}\right)=0
$$


which is equivalent to

$$
\left(c-z_{i}+\zeta d\right) d x_{i} d z_{i}+x_{i} d z_{i}^{2}=\rho d z_{i}^{2} .
$$

Now the vector $\mathbf{i}^{\prime}$ is always chosen for each intersection point in such a way that $\rho=\mathbf{e} \cdot \mathbf{i}^{\prime}$ is positive, and as a consequence it can be verified that equation 27 is not satisfied by false cone solutions. In practice the most difficult cases arise when the semi-cone angle $\alpha$ is near 90 degrees, and the tolerances become severe.

If the direction vector $\Omega$ lies in a plane through a containing the Z-axis, the condition being $\mathbf{a} \cdot \Omega \times \mathbf{k}=a \eta-b \xi=0$, the problem becomes that of finding the intersection of the given line with other straight lines (cone elements) in this plane. It can be readily shown that the distances from a to the two possible intersecting points with the cone elements are given by

$$
\begin{aligned}
d & =\frac{\left(z_{0}-c\right) \cos \phi \tan \alpha+\sqrt{a^{2}+b^{2}}}{\zeta \cos \phi \tan \alpha-\Omega \cdot \mathbf{P}} \\
\cos \phi & = \pm 1
\end{aligned}
$$

where

$$
\begin{aligned}
\mathbf{a} & =a \mathbf{i}+b \mathbf{j}+c \mathbf{k}=\sqrt{a^{2}+b^{2}} \mathbf{P}+c \mathbf{k} \\
\mathbf{P} & =\frac{a \mathbf{i}+b \mathbf{j}}{\sqrt{a^{2}+b^{2}}} \\
\mathbf{\Omega} & =(\boldsymbol{\Omega} \cdot \mathbf{P}) \mathbf{P}+\zeta \mathbf{k} \\
z_{0} & =z_{i}-x_{i} \cot \alpha \\
\tan \alpha & =\frac{d x_{i}}{d z_{i}}
\end{aligned}
$$

False solutions can be discarded as described previously.

\section{The Subroutine WHERE}

The subroutine WHERE calculates the identity of the mesh cell (cell number or number pair) within which a point with given coordinates is located. WHERE carries out a systematic search procedure beginning with some cell in which the point is surmised to be located; the test based on equation 25 is used, although it is applied to triangles rather than quadrilaterals.

Consider a mesh quadrilateral in the XZ-plane with vertices $\mathbf{x}_{\mathbf{i}}(\mathrm{i}=1,2,3,4)$, and corresponding coordinates $\left(x_{i}, z_{i}\right)$. The image point $P^{\prime}$ (position vector $\mathbf{a}^{\prime}$ ) corresponding to the given point $P$ (position vector $\mathbf{a}=a \mathbf{i}+b \mathbf{j}+c \mathbf{k}$ ) has coordinates $(\rho, c)$, where $\rho=\sqrt{a^{2}+b^{2}}$. Construct the diagonal of the quadrilateral, $\mathbf{x}_{\mathbf{1}}-\mathbf{x}_{\mathbf{3}}$, and form the product $D_{13}=\left(\mathbf{x}_{\mathbf{1}}-\mathbf{x}_{\mathbf{3}}\right) \times\left(\mathbf{a}^{\prime}-\mathbf{x}_{\mathbf{3}}\right) \cdot \mathbf{j}$. If $D_{13}$ is negative, $\mathbf{a}^{\prime}$ lies outside triangle $1,3,4$; then, if 


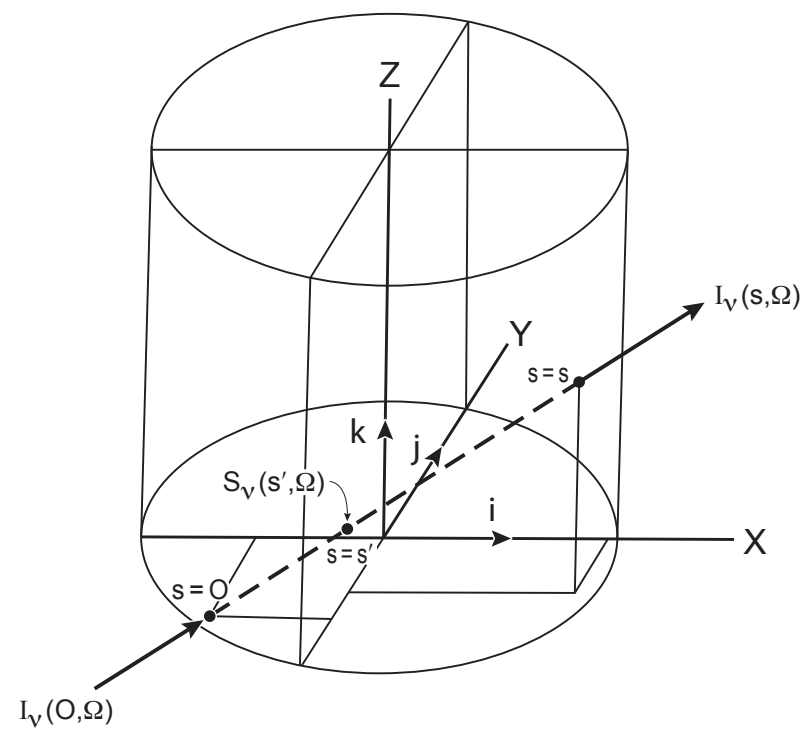

Figure 3: The incident, $I_{\nu}(0, \Omega)$, and emergent, $I_{\nu}(s, \Omega)$, intensities.

$D_{23}=\left(\mathbf{x}_{\mathbf{2}}-\mathbf{x}_{\mathbf{3}}\right) \times\left(\mathbf{a}^{\prime}-\mathbf{x}_{\mathbf{3}}\right) \cdot \mathbf{j}$ is negative, $P^{\prime}$ lies outside the quadrilateral, and the search proceeds to the adjoining cell that also contains side 3,2 . If $D_{23}$ is positive, it is necessary to form $D_{12}=\left(\mathbf{a}^{\prime}-\mathbf{x}_{\mathbf{1}}\right) \times\left(\mathbf{x}_{\mathbf{2}}-\mathbf{x}_{\mathbf{1}}\right) \cdot \mathbf{j}$; if $D_{12}$ is negative, $P^{\prime}$ lies outside, and the search proceeds to the adjoining cell that also contains side 1,2 . If $D_{13}$ is positive, then the product $D_{34}=\left(\mathbf{a}^{\prime}-\mathbf{x}_{\mathbf{3}}\right) \times\left(\mathbf{x}_{\mathbf{4}}-\mathbf{x}_{\mathbf{3}}\right) \cdot \mathbf{j}$ is formed, and finally $D_{14}=\left(\mathbf{a}^{\prime}-\mathbf{x}_{\mathbf{4}}\right) \times\left(\mathbf{x}_{\mathbf{1}}-\mathbf{x}_{\mathbf{4}}\right) \cdot \mathbf{j}$, if required.

\section{The Calculation of Isophotes}

Consider the problem of calculating the emergent radiance field for a low-altitude nuclear explosion. The geometric form of such a fireball is initially spherical at very early times, evolving later to a toroid with a vertical axis. Radiation-Hydrodynamic codes using cylindrical geometry have been written at Los Alamos that compute the evolution of such explosions. At each time step in the evolution, the physical variables, temperature, density, hot-air absorption coefficients, etc., are calculated for each mesh cell. Such information is stored only for certain preselected times and is later recovered for postprocessing. The program ISOPHOT, given in the appendix, is usually run in this mode. The radiance calculations can be accomplished with the same method of radiative transfer used in evolving the fireball, such as discrete ordinates; however, because HOWFAR is available to calculate the slant distance, s, through a mesh cell, the formal solution of the equation of transfer can be easily applied and should give the most accurate results possible with the given constraints created by the grid structure. Furthermore this gives an independent check on the transfer method adopted for the evolution computation. The 
emergent radiation is given by (refer to Fig. 3):

$$
I_{\nu}(s, \boldsymbol{\Omega})=I_{\nu}(0, \boldsymbol{\Omega}) e^{-\int_{0}^{s} k_{\nu}\left(s^{\prime}\right) d s^{\prime}}+\int_{0}^{s} S_{\nu}\left(s^{\prime}, \boldsymbol{\Omega}\right) e^{\left(-\int_{s^{\prime}}^{s} k_{\nu}\left(s^{\prime \prime}\right) d s^{\prime \prime}\right)} k_{\nu}\left(s^{\prime}\right) d s^{\prime}
$$

where $I_{\nu}(0, \Omega)$ is the radiance of light of frequency $\nu$ in the direction $\Omega$ at $s=0, k_{\nu}\left(s^{\prime}\right)$ is the absorption coefficient at $s^{\prime}$, and $S_{\nu}\left(s^{\prime}, \Omega\right)$ is the source function at $s^{\prime}$ in direction $\Omega$. For thermal radiation the source function is the Planck function $B_{\nu}(T)$, and

$$
S_{\nu}\left(s^{\prime}, \boldsymbol{\Omega}\right)=B_{\nu}\left[T\left(s^{\prime}\right)\right]=\frac{2 h \nu^{3}}{c^{2}} \frac{1}{e^{h \nu / k T}-1}
$$

where $T$ is the absolute temperature. Equation 35 can be applied to a single mesh cell within which the source function is assumed to be constant, giving

$$
I_{\nu}(s, \boldsymbol{\Omega})=I_{\nu}(0, \boldsymbol{\Omega}) e^{-k_{\nu} s}+B_{\nu}(T)\left(1-e^{-k_{\nu} s}\right),
$$

where $T$ and $k_{\nu}$ are appropriate for the cell, $s$ is the slant distance through the cell calculated by $H O W F A R$ and $I_{\nu}(0, \Omega)$ the radiance incident on the cell in direction $\Omega$. This procedure can be applied along a chosen ray through the entire mesh, cell by cell. In order to obtain isophote contours, it is necessary to find emergent radiances in this fashion along many rays through the fireball to the observer's position (we routinely use about 1000 to 3000 rays). A contour-line plotting routine can then be used to produce isophote graphs. Finally, by integration over the field of isophotes the irradiance, $D_{\nu}$, at the observer can be found,

$$
D_{\nu}=\int_{0}^{\omega_{0}} I_{\nu} \cos \theta d \omega
$$

$I_{\nu}$ is the radiance emitted in the direction of the observer by a surface area element, $d \Sigma$, of the source, $\omega_{0}$ is the solid angle subtended by the source and $d \omega$ that subtended by $d \Sigma$ as viewed from the observer's position. Theta is the angle between the collimation axis of the camera, or photometer, and the axis of $d \omega$. Any convenient cubature formula can be used to evaluate the integral.

In the program ISOPHOT, given in the appendix, the following procedure is adopted (refer to Fig. 4). Let $\mathbf{a}_{\mathbf{0}}=a_{0} \mathbf{i}+c_{0} \mathbf{k}$ be the position vector of the observer in the XZ-plane with respect to the initial center, $C$, of the mesh. The code first searches the mesh for the cell with the highest temperature. The vertex of this cell has coordinates $\left(x_{h}, z_{h}\right)$ in the XZ-plane. A line is drawn from the observer $\mathbf{a}_{\mathbf{0}}\left(a_{o}, c_{0}\right)$ to the point $C: \mathbf{H}\left(0, z_{h}\right)$ in the direction

$$
\Omega^{*}=\frac{\left(\mathbf{H}_{0}-\mathbf{a}_{\mathbf{0}}\right)}{\left|\mathbf{H}_{\mathbf{0}}-\mathbf{a}_{\mathbf{0}}\right|} .
$$

Next, a plane is constructed through the point $\mathbf{H}\left(0, z_{h}\right)$ perpendicular to $\mathbf{\Omega}^{*}$. A rectangular cartesian coordinate system (coordinates $y, l$ ) is formed in this plane with the origin at $\mathrm{C}$ and axes in directions $\mathbf{j}=\mathbf{k} \times \mathbf{i}$ and $\mathbf{l}=\mathbf{j} \times \mathbf{\Omega}^{*}$. A grid is created in the JL-plane from $y=0$ to $+R$ along the J-axis and from $l=-R$ to $R$ along the L-axis, where $2 R$ 


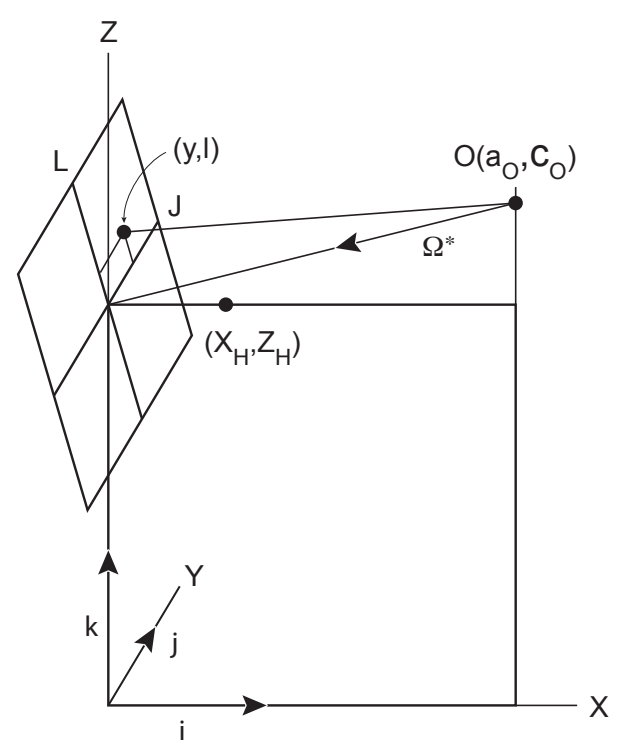

Figure 4: The observer, $\mathrm{O}$, is located at $\left(a_{0}, c_{0}\right)$.

is the maximum linear dimension of the fireball. The radiance values display symmetry with respect to the L-axis, because the physical parameters possess axial symmetry about the Z-axis. Therefore, it is only necessary to calculate radiances on the positive side of the L-axis; for this purpose, a grid can be used with $y_{j} / R=0,1, \ldots n(j=1, \ldots, N+1)$ and $l_{k} / R=-N,-N+1, \ldots-1,-, 1, \ldots, N(k=1, \ldots, 2 N+1)$. The integration to find the irradiance $D_{\nu}$ can be performed in two parts over positive and negative $l$ values: thus,

$$
D_{\nu}=\frac{2 A}{r_{H}^{2}} \Sigma_{j=1}^{N+1} \Sigma_{k=1}^{2 N+1} w_{j} w_{k} I_{j, k}(\nu) \cos ^{4} \theta_{j, k}
$$

where

$$
r_{H}=\left|\mathbf{H}-\mathbf{a}_{\mathbf{0}}\right|=\sqrt{a_{0}^{2}+\left(c_{0}-z_{h}\right)^{2}},
$$

$A$ is the area of a grid cell,

$$
\cos \theta_{j, k}=\frac{r_{H}}{\sqrt{y_{j}^{2}+l_{k}^{2}+r_{H}^{2}}},
$$

and $I_{j, k}$ is the radiance in the direction of the observer at point $\left(y_{j}, l_{k}\right)$, and $w_{j}, w_{k}$ are appropriate quadrature weights.

Fig. 5 shows the isophotes computed in the fashion just described for a nominal 10-kt near-surface (altitude $200 \mathrm{~m}$ ) nuclear explosion at the evolution time of 2 seconds. The fireball is beginning to develop into the form of a ring-shaped vortex, or toroid, under the action of the ground reflected shock. The observer is located at a large distance horizontally from the fireball. The two points of maximum radiance are labelled $O$, 


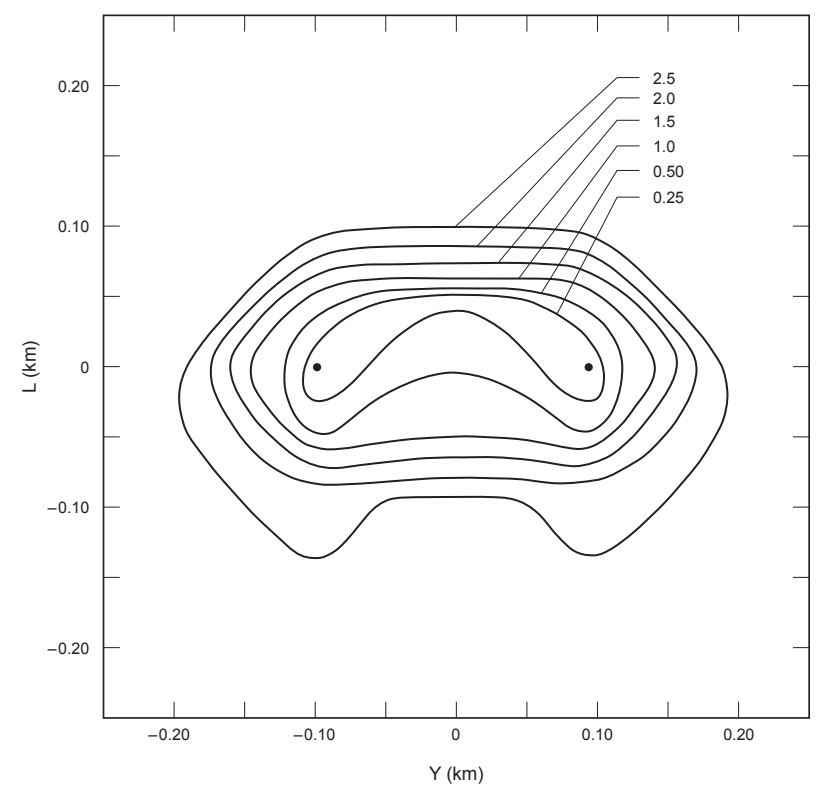

Figure 5: Isophotes for $10 \mathrm{kt}$ with 200-m HOB at 2 s.

and the isophote contours are assigned relative values in the stellar-magnitude scale, $m=-2.5 \log [I(m) / I(0)]$, where $m$ is is the magnitude associated with radiance $I(m)$, and $I(0)$ is the maximum radiance arbitrarily assigned magnitude zero.

\section{Current Work}

\subsection{Preliminaries}

In the work here $I_{\nu}, B_{\nu}$, and $S_{\nu}$ all have units of energy per unit area, per unit time, per steradian, and per $\mathrm{Hz}$ and have names of specific monochromatic intensity, the Planck function and the source function, respectively. The term radiance is also used for $I_{\nu}$. The irradiance, $D_{\nu}$, is the integral of $I_{\nu}$ over solid angle and has the units of a flux, energy per unit area, per unit time, per Hz. The intensity, $I_{\nu}$, is the basic dependent variable in the radiative transfer equation.

In the late 1970s, the computational approach had been incorporated into the existing atmospheric effects code SnYAQUI that combined the finite difference hydrodynamic code YAQUI, Amsden and Hirt, (1973), with the discrete ordinate Sn algorithm for radiative transfer, (Lathrop, 1972, Lathrop and Brinkely, 1973). Over time, as computational resources shifted from a central computing facility with large mainframes, to distributed computing with increasingly powerful desktops, we have migrated our atmospheric effects codes. Along the way, we adopted the newer hydro package CAVEAT, Addessio et al. (1992) and coupled it to the Sn discrete ordinate code to make SnCAVEAT. In making 
SnCAVEAT we included some new developments in the Sn method, Hill and Patternoster (1982). SnCAVEAT now runs on Sun Solaris Unix, with the $f 90$ compiler; Mac OS X, with the Absoft and g95 compilers; and a Los Alamos National Laboratory high performance computing cluster, with the Portland Group $f 90$ compiler.

The code SnCAVEAT is a 2-D program usually run in cylindrical geometry, (r, z). Not every calculation of interest needs 2-D; often a 1-D program can be used. Once the ground surface is encountered or when bouyant deformation begins, then a calculation must transition from 1-D to at least 2-D. (One could argue that everything should be 3-D, but we are not there yet.) A strategy often followed is to perform the calculation with a 1-D, spherically symmetric, code until just before 2-D effects begin. Then one maps the 1-D problem into the the 2-D mesh and continues the evolution with the 2-D code. The same equation of state and multi-group opacity tables are used for the 1-D and 2-D codes. Our 1-D code is based on the work of Zinn (1973) as programmed in the code HYCHEM. Additional information on the code can be found in the other Zinn references in the bibliography, as well as Symbalisty et al. (1995). Another version of the 1-D code, called RADFLO, is described in Horak and Kodis (1983). RADFLO does not include the detailed atmospheric chemistry capability found in HYCHEM; otherwise, they are quite similar.

The Appendix provides a sample input file, a code listing, and two sample makefiles. There are some parts of the Isophote processor that are specific to our SnCAVEAT program. For use with outputs from other numerical applications, a few comments may be helpful. One needs the numerical mesh coordinates, the specific internal energy (energy/mass), the mass density (mass/unit volume), opacity data, planck functions and equation of state (EOS) data. In our application, the coordinates are in the variable $x v$, specific internal energy in sie, mass density in $r h o$, opacity data in $u k$, Planck functions in $p l b$ and equation of state data in $g t$ and $f p$. In the listing, the include files comdeck and comdeck1 hold a number of SnCAVEAT variables including those needed by the isophote code. These would be different with other codes, and the details of reading the binary dumps would be different as well. In the listing, common block rlc1 holds the opacity and planck functions while block state holds the equation of state (EOS) data.

The details of the EOS and opacity data can be found in Symbalisty et al. (1995) and Horak and Kodis (1983); however, we will provide a brief summary here. Let $T$ be temperature in ev, $P$ be pressure, $E$ be specific internal energy and $\rho$ mass density. Then $g t$ holds $T / E$ as a function of $E$ for 100 temperatures and seven densities. The array $f p$ holds $P /(\rho E)$ as a function of $E$ for 100 temperatures and 7 densities. The array

$u k$ holds the opacity data as Rosseland means, $\mathrm{cm}^{2} / \mathrm{gm}$, for 51 frequency bands, for 100 temperatures and seven densities.

\subsection{Isophote Application}

An isophote post processor was written that processes a SnCaveat binary dump file. This post processor follows the algorithm documented in Sections $1-5$. With the post 


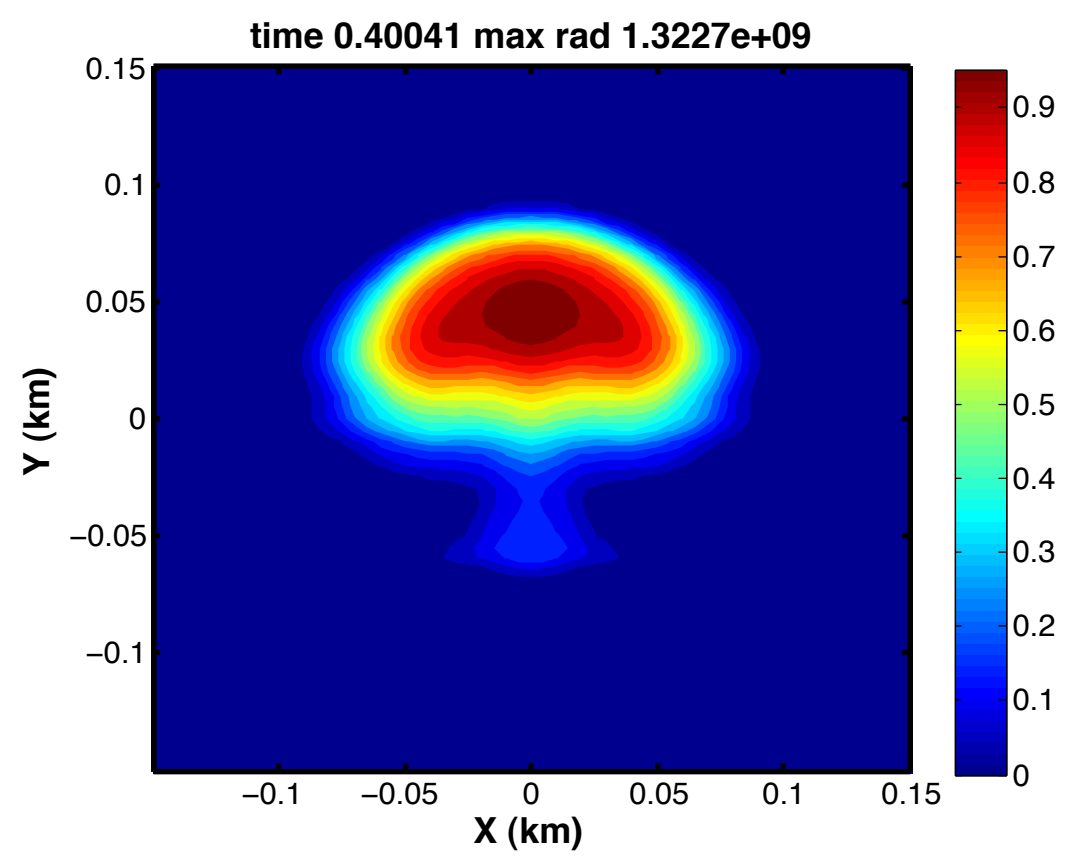

Figure 6: Isophotes for a 1-kt burst at 50-m HOB and a time of $0.4 \mathrm{~s}$, horizontal path

processor code, one can easily calculate contours for a series of SnCAVEAT dump files and change the observer look angle. The first example of the current version is given in Fig. 6 for a - kt burst at 50-m HOB with a horizontal look angle, at a nominal distance of $20000 \mathrm{~km}$, while Fig. 7 is the same except for a $45^{0}$ look angle. In these plots, the time and maximum radiance are given at the top of the plots. The contours are for radiance values normalized by the maximum radiance. The calculation was initialized at $1.5 \mathrm{~ms}$ from a 1-D HYCHEM run. Fig. 8 then gives the Si irradiance (Eq. 43) as a function of time for the $45^{0}$ look angle.

For a given observer position, and a given set of isophote contours, Eq. 38, for $D_{\nu}$ gives the energy per unit area, per unit time (a flux), at the observer position. One could then, for some detector with some area A, determine the total energy per second in the detector by $A D_{\nu}$.

The irradiance values in the different bands can be summed with appropriate weights to calculate specific responses. For a silicon detector, we determine the irradiance with the following summation:

$$
\begin{aligned}
\operatorname{siirad} & =0.17 * h \operatorname{kirad}(7)+0.95 * h \operatorname{kirad}(8)+0.89 * \operatorname{hkirad}(9) \\
& +0.62 * h \operatorname{kirad}(10)+0.37 * h \operatorname{kirad}(11)+0.12 * \operatorname{hkirad}(12)
\end{aligned}
$$




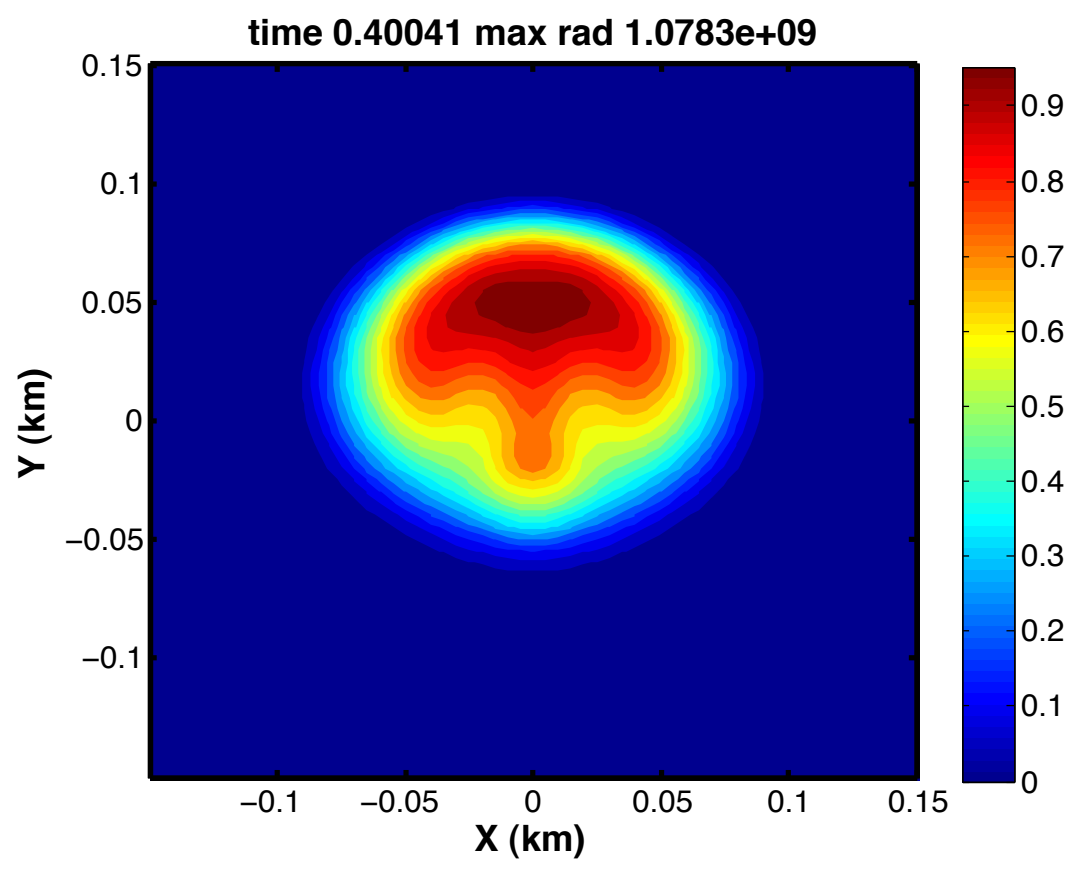

Figure 7: Isophotes for a 1-kt burst at 50-m HOB and a time of $0.4 \mathrm{~s}$, and $45^{\circ}$ path. 


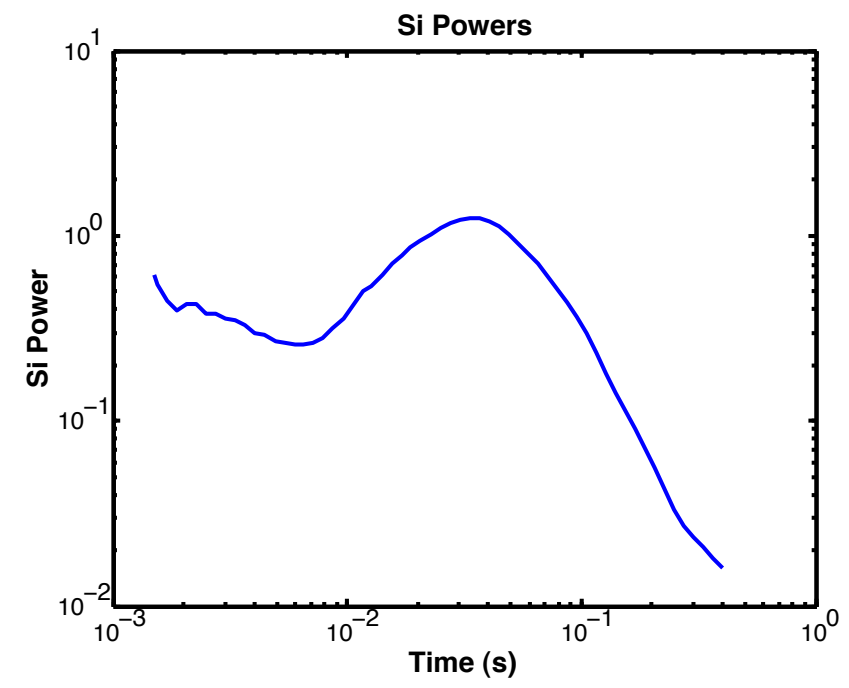

Figure 8: The Si band power vs time curve for $45^{0}$ look angle.

where the hkirad values are the fluxes in the bands 7 to 12 , and the coefficients give the relative weight for calculating the bhangmeter-weighted Si band. This is what is plotted in Fig 8. Although siirad has the units of a flux, it could also be viewed as the power in a unit area.

\subsection{Particular Cases}

One reason for using a post-processing code, such as isophote, is to calculate fluxes from different look angles (expressed as either elevation angle or zenith angle). For lower heights of burst (HOB), where there is ground interaction, 2-D effects become important, and the fireball evolution departs from that for a 1-D free air burst. For a burst on an ideal perfectly reflecting surface, $\mathrm{HOB}=0$, where downward-directed energy is reflected back into the fireball, there is a well known factor of two effect on the observed yield, for blast as well as optical. On the other hand, for a higher HOB, one would expect a 1-D free-air behavior with a 2-D code.

We have performed a series of calculations of a 10-kt burst at HOBs of (meters): 0, 30, 60, 120, and 240. These were chosen in part to test for the surface burst factor of two in yield as measured by minimum time in the silicon power time curve. The fireball evolution was performed with our 2-D code SNCAVEAT. Binary mesh dumps are made periodically over the course of the calculation. The isophote post-processor then reads the dumps and performs the radiative transport through the fireball structure. These calculations were performed with Sn order 4. A new initialization scheme was used in SnCAVEAT that follows the philosophy that is used in our 1-D code, HYCHEM, Zinn 


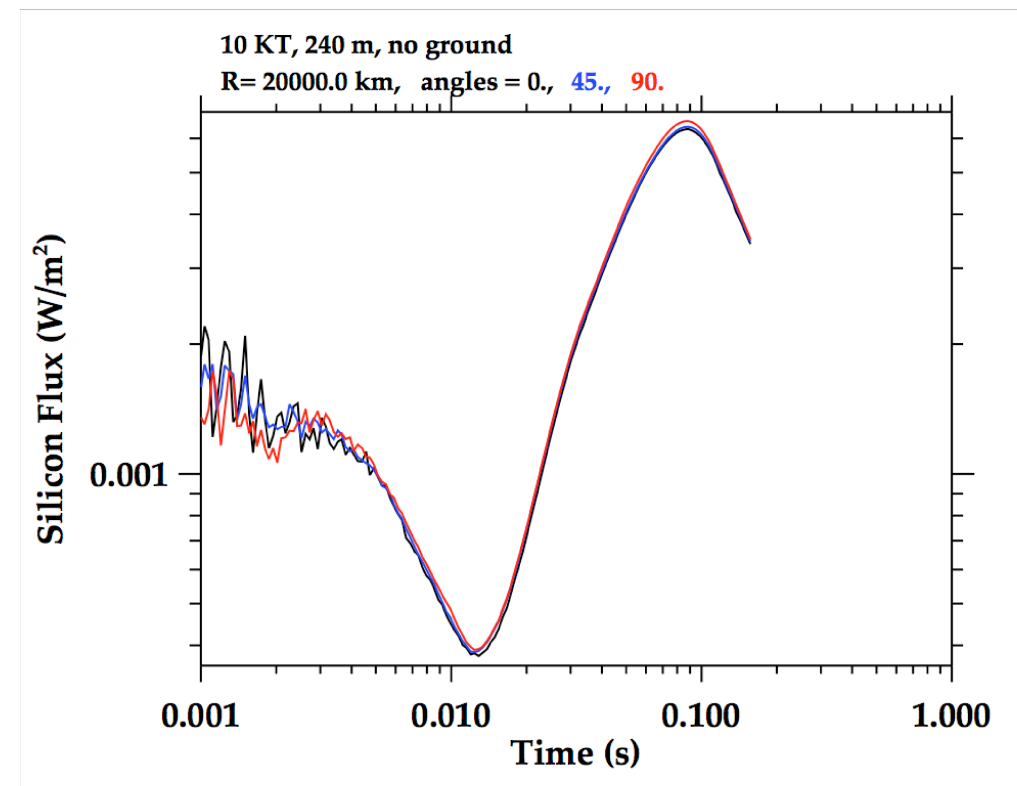

Figure 9: Si flux versus time for the 10 kt, 240-m HOB at three elevation angles.

(1973). In the following figures we show data from the isophote post processor for an observer at $20000 \mathrm{~km}$ and at various elevation angles.

In Fig. 9, for the 240-m HOB, we show Si-weighted fluxes for the elevation angles of $0^{0}, 45^{\circ}$ and $90^{\circ}$. In this calculation, there was no ground in the calculation. As expected the curves overlay nicely, with a slight difference at second max, showing no look angle dependence, the expected result for no ground interaction. Next, in Fig. 10 for the 0-m HOB we show Si fluxes at three elevation angles. Here, the ground interaction is immediate, the fireball structure is not the same as seen from different angles, and the large differences in the Si flux curves can be understood by reference to the isophote contours. In Fig. 11, for the 0-m HOB, we first show the contours for the horizontal look angle, followed by Fig. 12 for the vertical look angle. Both are plotted on the same scale, and the integration over the contours of Fig. 12 will clearly give a larger value than that for Fig. 11.

The HOB effect on minimum time is illustrated in Fig. 13 where we show Si flux versus time for the 240-m HOB and the 0-m HOB, both with a horizontal look angle. In this figure, we also show one curve from the Los Alamos version of Hychem. The surface burst has a later minimum time than the 240-m HOB, $0.0149 \mathrm{~s}$ as opposed to 0.0120 s. The value for the $0 \mathrm{~m} \mathrm{HOB}$ is exactly that for a free air 20-kt burst as given by the scaling law in Symbalisty et al. (1995). This demonstrates the HOB effect rather nicely for optical parameters. The 240-m values are larger consistent with the contours shown in Figs. 11 and 12. The early time, through first maximum, radiative output is difficult to calculate because of the large optical depths in the very hot shock that would require 


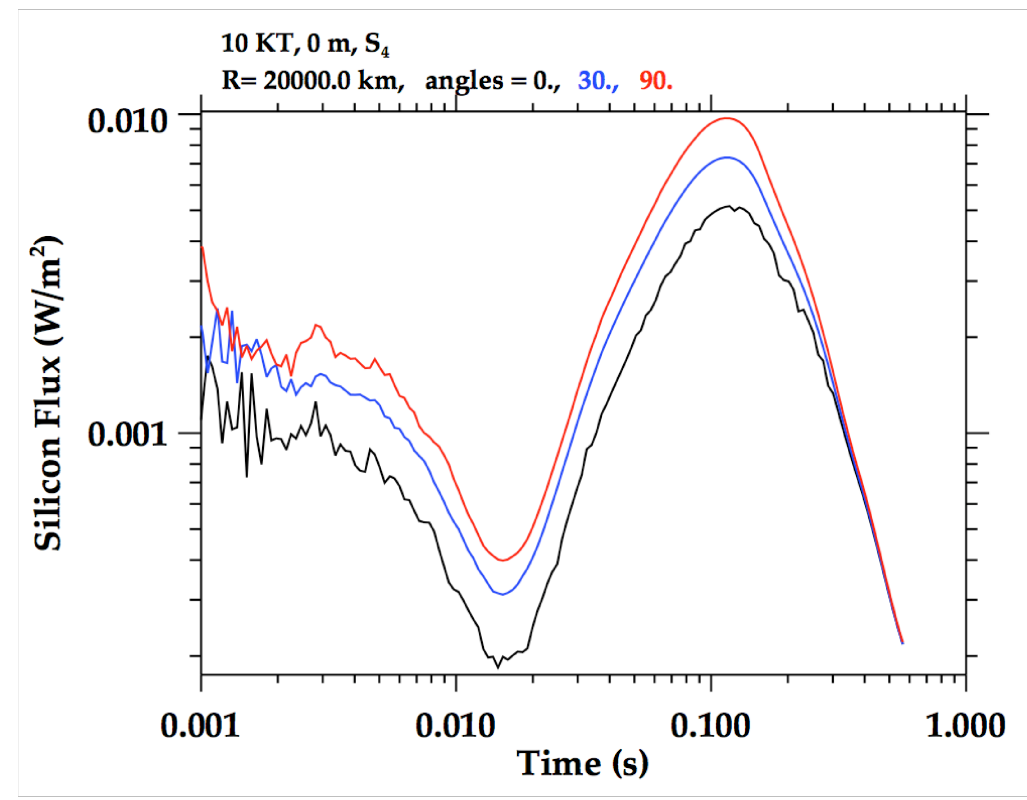

Figure 10: Si flux versus time for the $10 \mathrm{kt}, 0-\mathrm{m}$ HOB at three elevation angles.

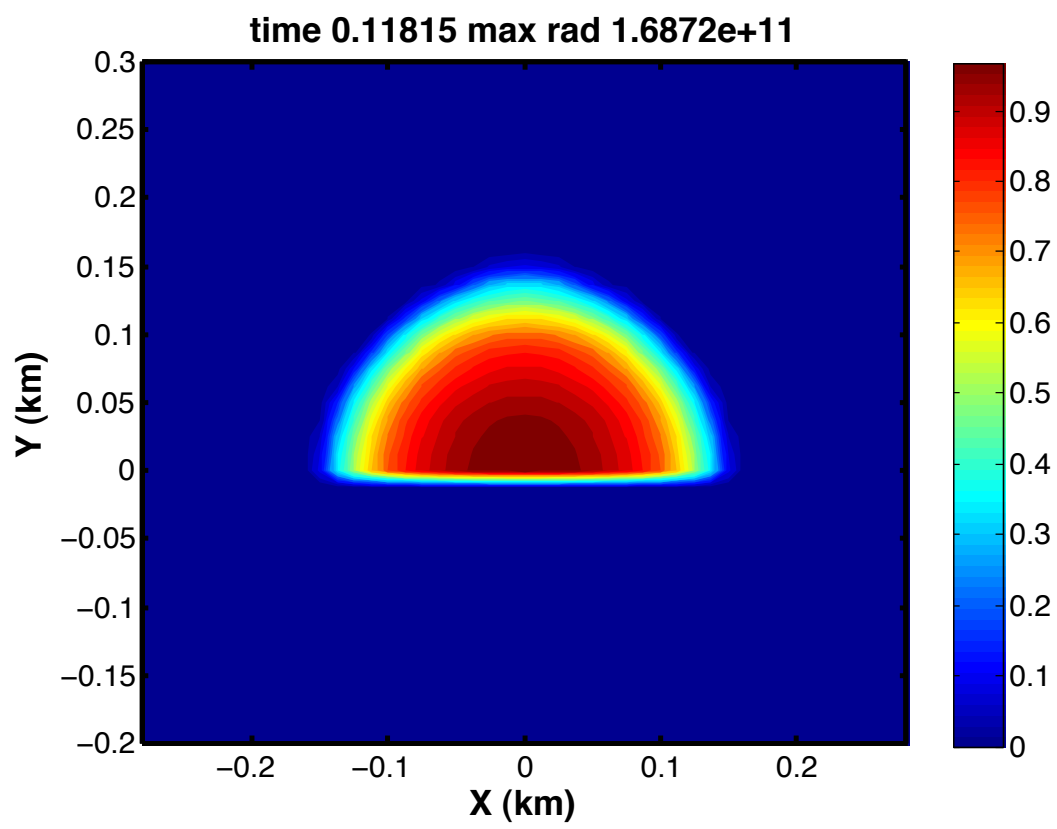

Figure 11: Isophotes for a $10 \mathrm{kt}$ burst at $0-\mathrm{m}$ HOB and $0^{0}$ elevation angle. 


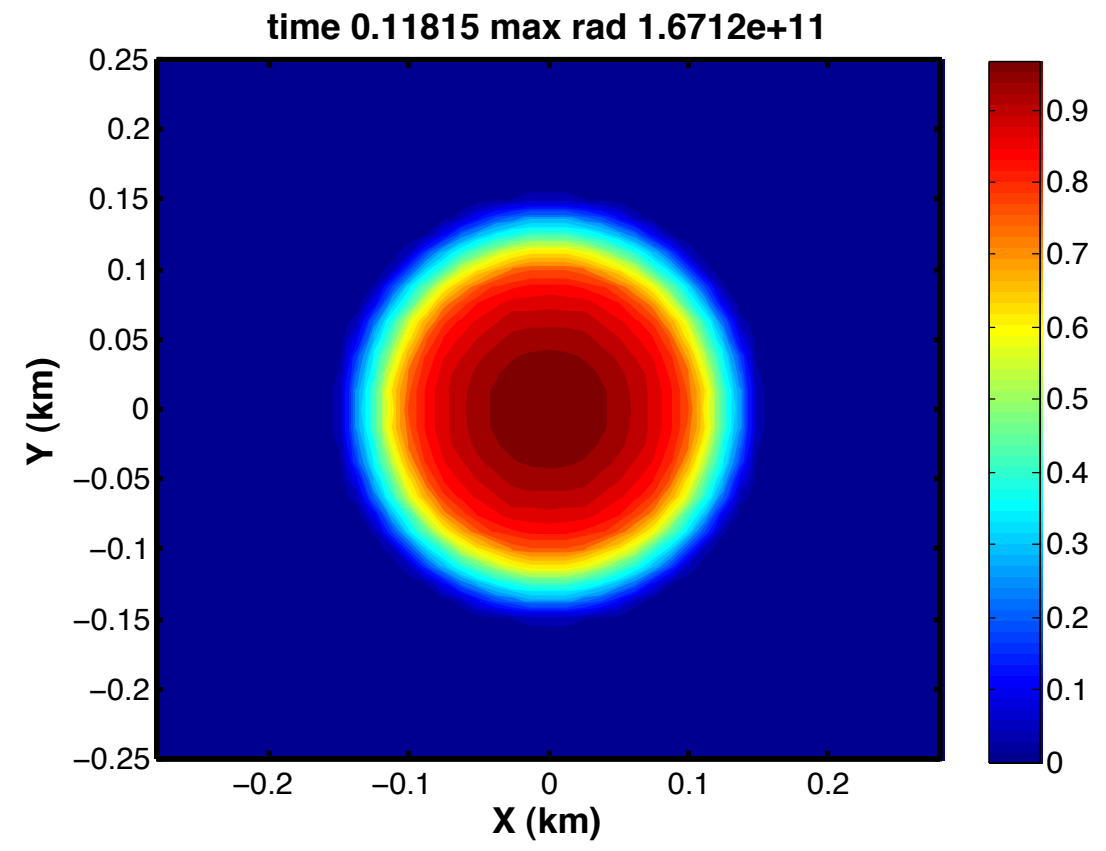

Figure 12: Isophotes for a 10-kt burst at 0-m HOB and $90^{\circ}$ elevation angle. 
prohibitively small cells. In this regime, HYCHEM uses tables of shock brightness versus shock speed obtained from steady state shock theory, Symbalisty et al. (1995) and Zinn and Sutherland (1981). This avoids the oscillations in brightness such as are shown in the early part of the SnCAVEAT curves until the radiative scales are compatible with the cell sizes.

Table 1 gives the full set of minimum times for the different HOB calculations.

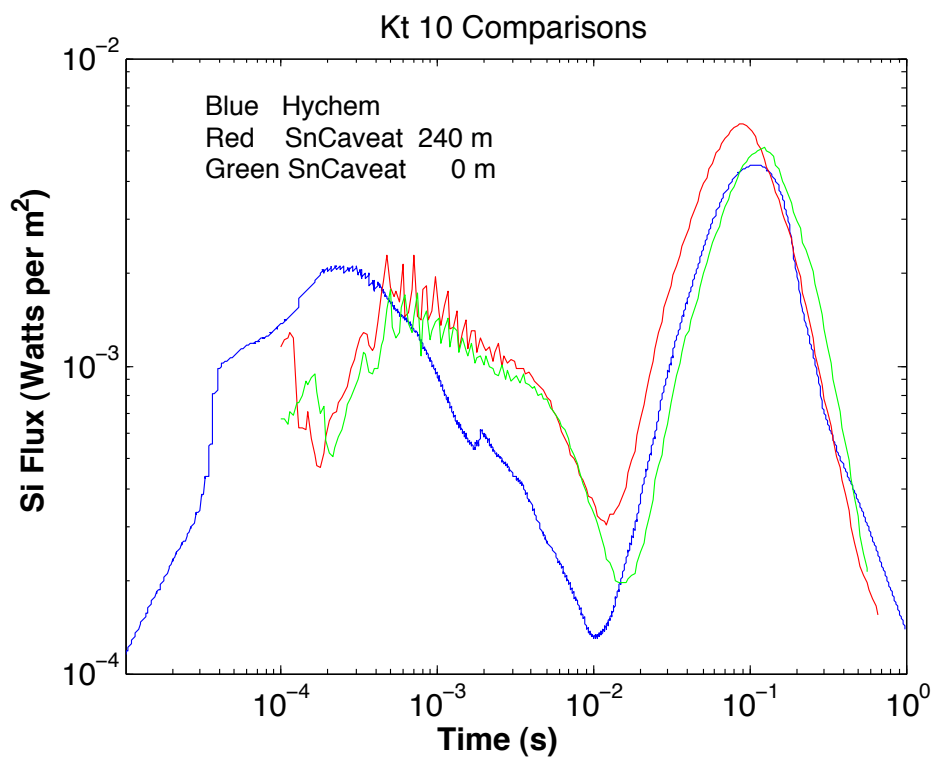

Figure 13: Si flux versus time for the $10 \mathrm{kt}, 0-\mathrm{m}$ and 240-m HOB showing the delayed minimum time for the $0-\mathrm{m}$ HOB case.

Table 1: Minimum times for the 10-kt HOB series.

\begin{tabular}{cc}
\hline $\operatorname{HOB}(\mathrm{m})$ & $\mathrm{T} \min (\mathrm{s})$ \\
\hline 0 & 0.0149 \\
30 & 0.0133 \\
60 & 0.0121 \\
120 & 0.0120 \\
240 & 0.0120 \\
\hline
\end{tabular}




\section{Summary}

In this report, we have illustrated the application of our isophote post-processor program, isopost.f, on some SnCaveat 2D fireball simulations. In addition, we have shown the delay in minimum time, compared to a free air burst, for lower heights of burst, including a surface burst. We recover the well known factor of two in apparent yield for the surface burst. The current version does require a cylindrical $(\mathrm{r}, \mathrm{z})$ mesh. With this constraint, the code could be applied to other CFD code outputs with some changes in reading in the CFD output and having an appropriate set of opacity and equation-of-state data.

A similar and independent capability was developed at Sandia National Laboratory and is discussed in Dreike et al. (2006).

\section{Acknowledgements}

The original draft report was prepared by Henry Horak and John W. Kodis (deceased). Much of the supporting work was done by several individuals working on atmospheric nuclear effects in various groups within LANL. In addition to Horak and Kodis, important contributions to the overall effort in effects work were made by Brook Sanford, Eric Jones, Richard Anderson, and John Zinn. We have benefited from productive collaboration with the LANL numerical hydrodynamics group, T-3. Tom Hill made significant contributions in ensuring that our Sn radiative transfer algorithms were efficiently incorporated and in assisting with new applications. Christy Flaming prepared the figures in Sections 1-5. We thank Laurie Triplett for reviewing the manuscript. Support, over the years, has come from the Defense Nuclear Agency (now the Defense Threat Reduction Agency), the Department of Energy, the Air Force and LANL. 


\section{Bibliography}

1. Addessio, Frank L., John R. Baumgardner, John K. Dukowicz, Norman L. Johnson, Bryan A. Kashiwa, Rick M. Rauenzahn and Charles Zemach, CAVEAT: A Computer Code for Fluid Dynamics Problems with Large Distortion and Internal Slip, Los Alamos National Laboratory technical report LA-10613-MS, Rev. 1 (1992).

2. Amsden, A. and C. Hirt, YAQUI: An Arbitrary Lagrangian-Eulerian Computer Program for Fluid Flow at All Speeds, Los Alamos Scientific Laboratory report LA5100 (1973).

3. Anderson, R. and M. Sandford II, YOKIFER: A Two-Dimensional Hydrodynamics and Radiation Transport Program, Los Alamos Scientific Laboratory report LA5704-MS (1974).

4. Dreike, Philip L., Raymond L. Bell, Gary D. Cable, William Hilbun, Michael L. Hobbs, Anne R. Moats, J. Randy Weatherby and Robert J. Weir, Progress on Modeling Optical Signals from Nuclear Detonations in Multi-Dimensional Emplacements, Sandia National Laboratory report SAND2006-7510 (2006). (Report is OUO.)

5. Hill, Thomas R. and Richard R. Paternoster, Two-Dimensional Spatial-Discretization Methods on a Lagrangian Mesh, Los Alamos National Laboratory technical report, LA-UR-82-1055 (1982).

6. Horak, H., E. Jones, J. Sandford II, R. Whitaker, R. Anderson, and J. Kodis, Two-Dimensional Radiation Hydrodynamic Calculations for a Nominal 1-Mt Nuclear Explosion Near the Ground, Los Alamos National Laboratory report, LA-9137 (1982).

7. Horak, H.G. and J. Kodis, RADFLO - A User's Manual, Los Alamos National Laboratory report LA-9245-M May (1983).

8. Lathrop, K.D., Discrete-Ordinates Methods for the Numerical Solution of the Transport Equation, Reactor Technology, 15, 107-133 (1972).

9. Lathrop, K. and F. Brinkley, TWOTRAN II: An Interfaced, Exportable Version of the TWOTRAN Code for Two-Dimensional Transport, Los Alamos Scientific Laboratory report LA-4848-MS (1973).

10. Symbalisty, E.M.D., J. Zinn, and R.W. Whitaker, RADFLO Physics and Algorithms, Los Alamos National Laboratory report LA-12988-MS (1995).

11. Zinn, J., A Finite Difference Scheme for Time-Dependent Spherical RadiationHydrodynamics Problems, J. Comput. Phys., 13, 569 (1973). 


\section{Appendix}

\subsection{Sample input file}

Below we show the input file for running the isophote code that is namelist based.

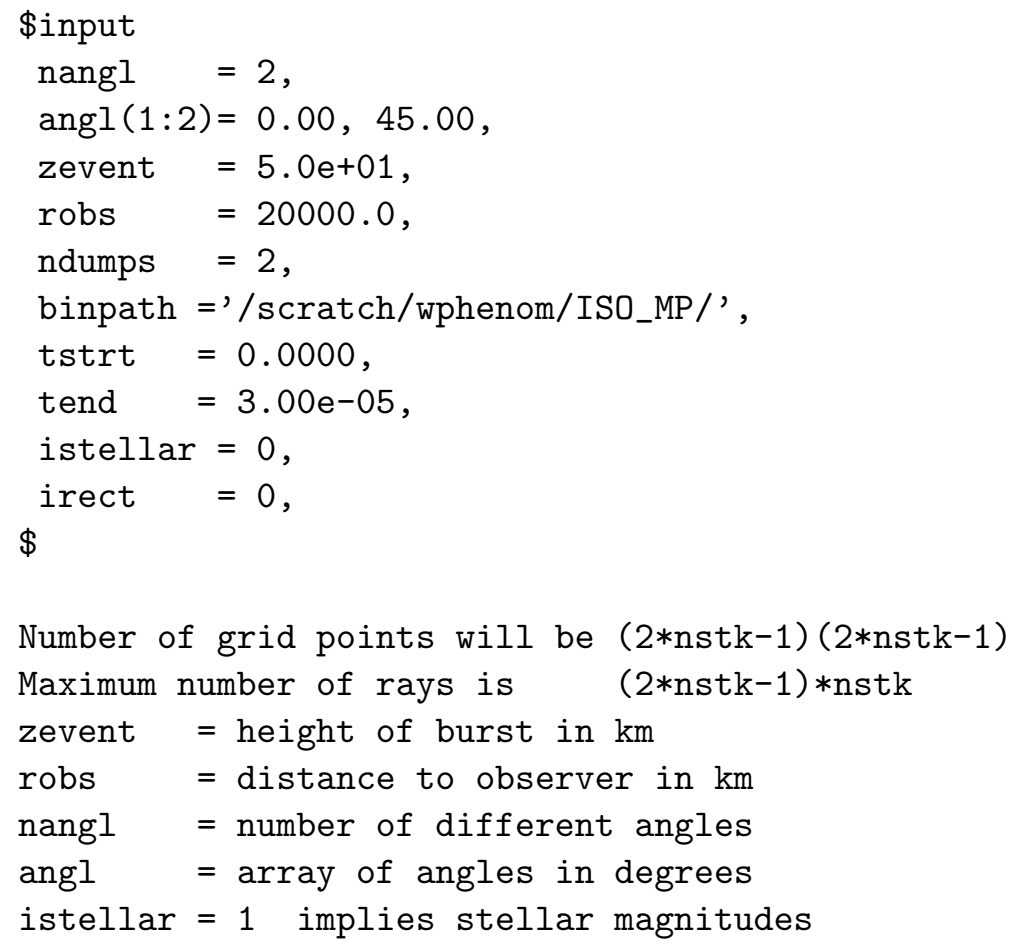




\subsection{Code listing}

Now we provide a listing of the isophote processor. This version does incorporate OpenMP for shared memory, multi-processor machines. The OpenMP instructions appear as compiler directives that are just seen as comments for a serial processor computer.

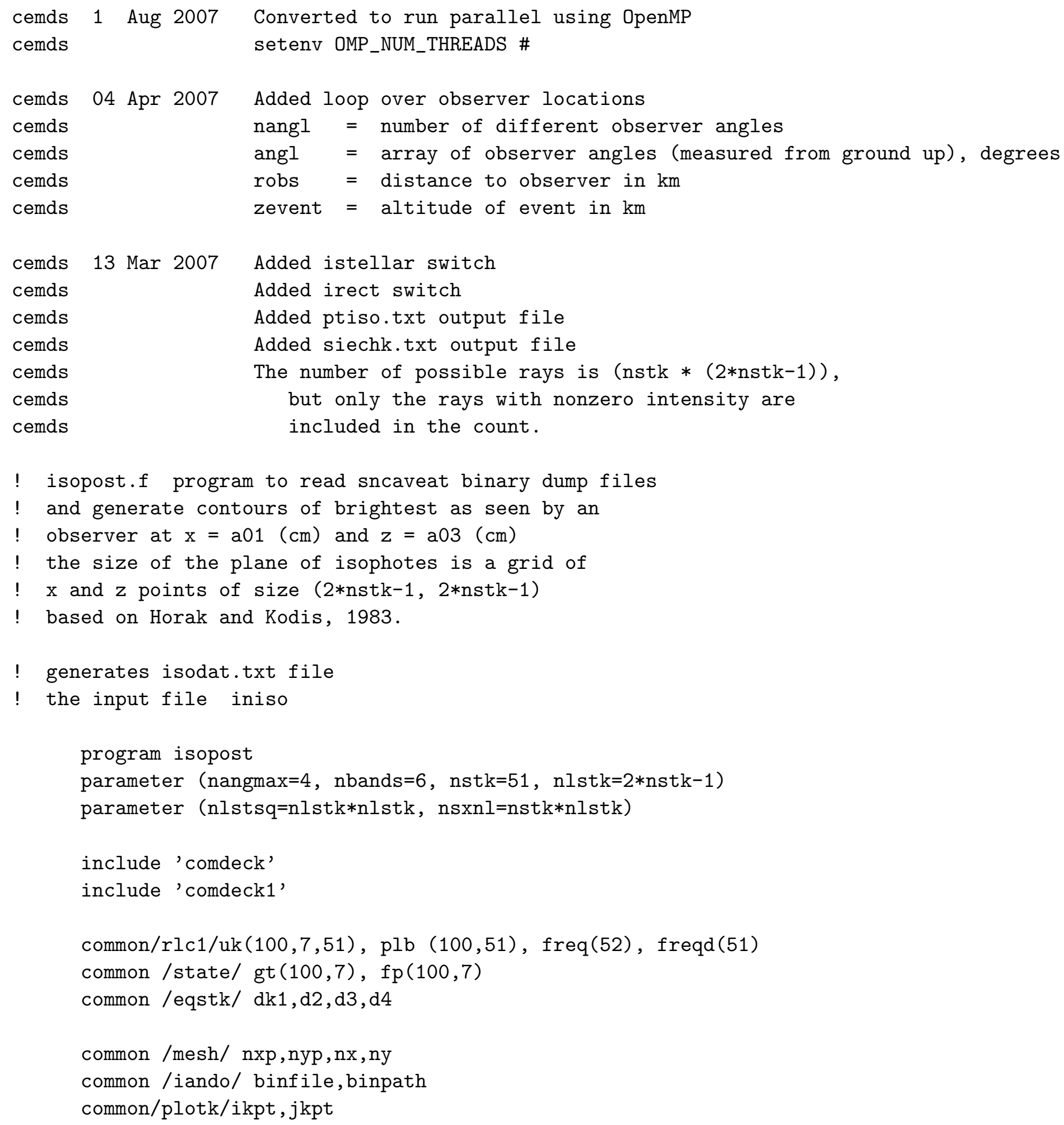




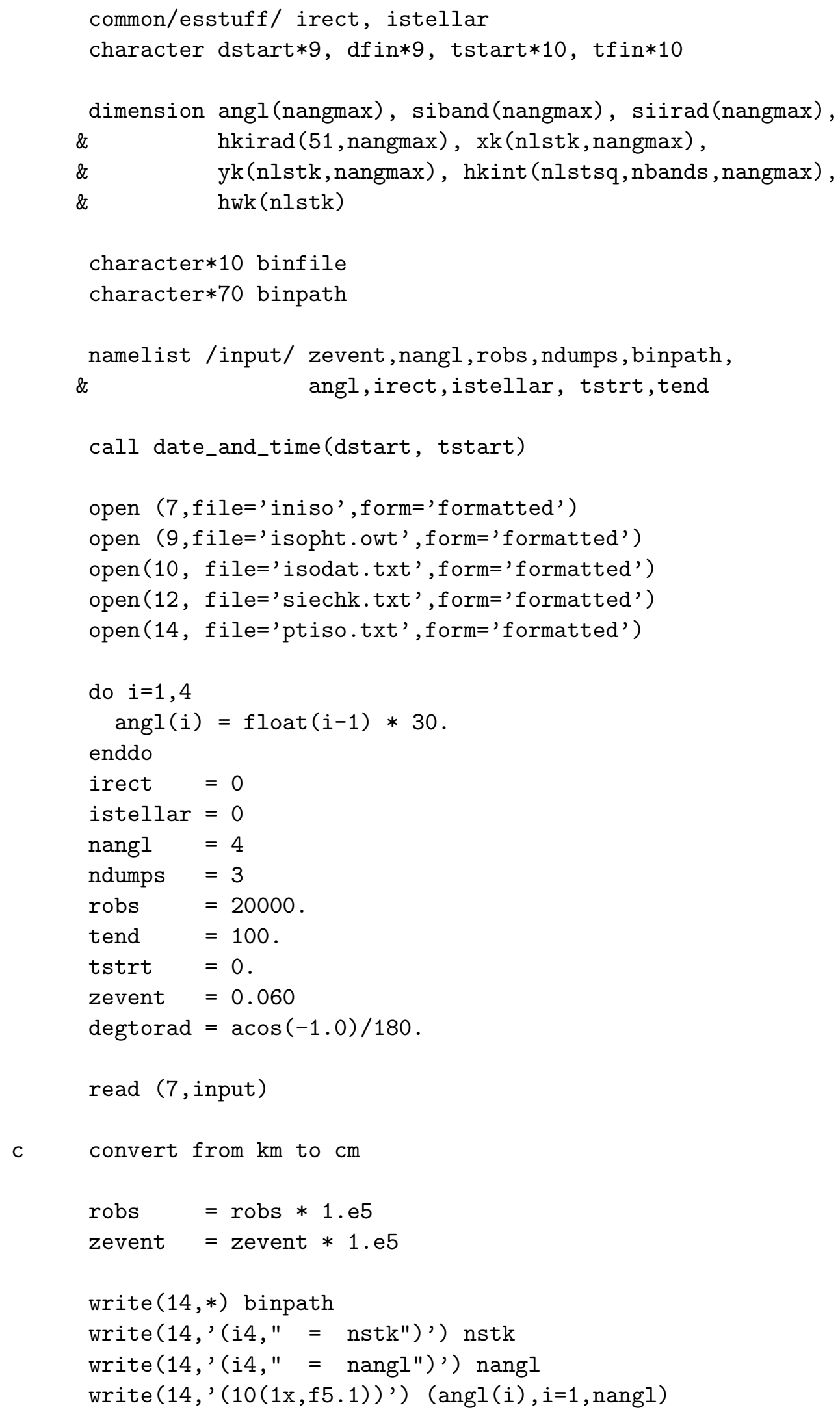




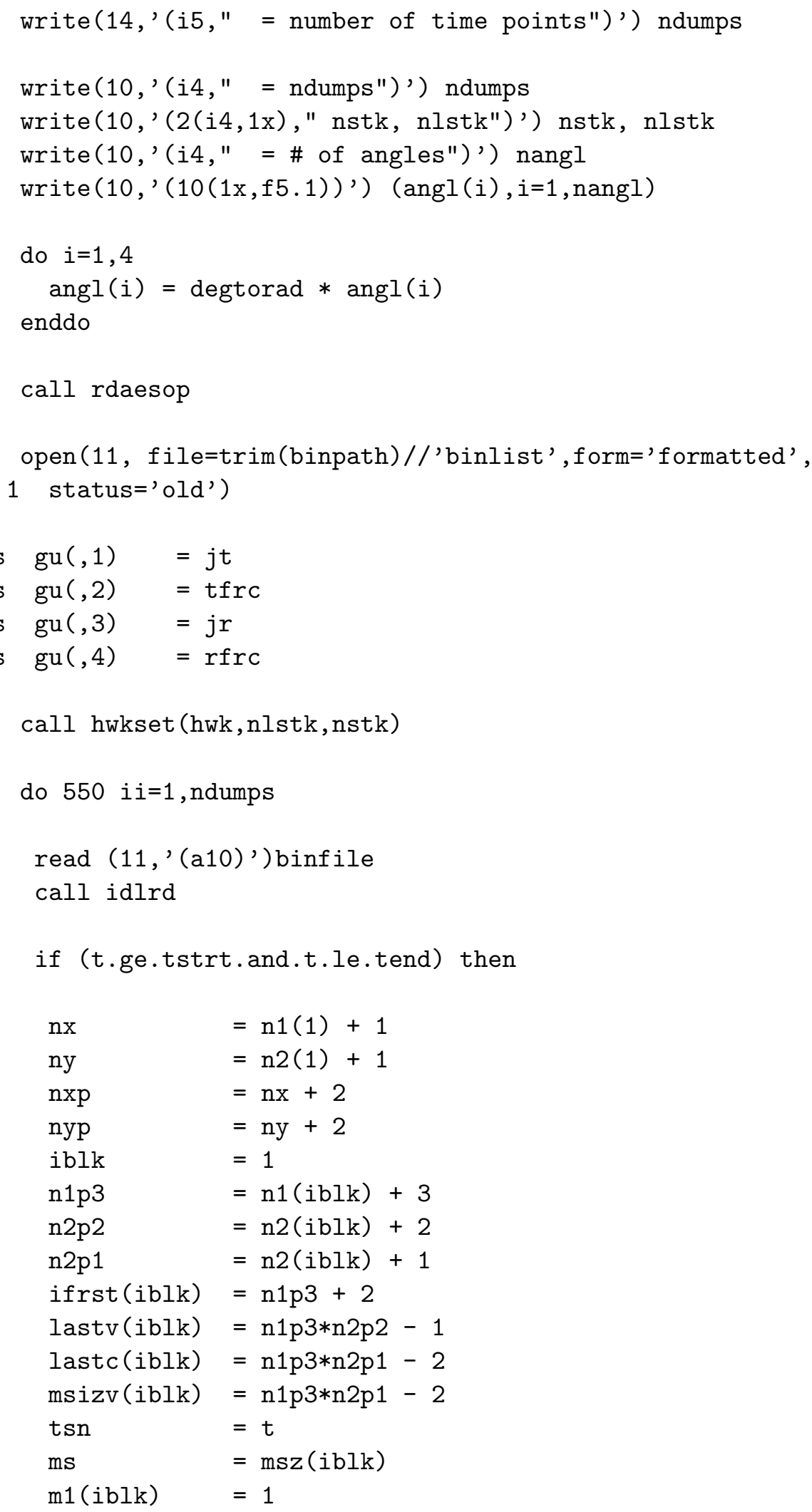




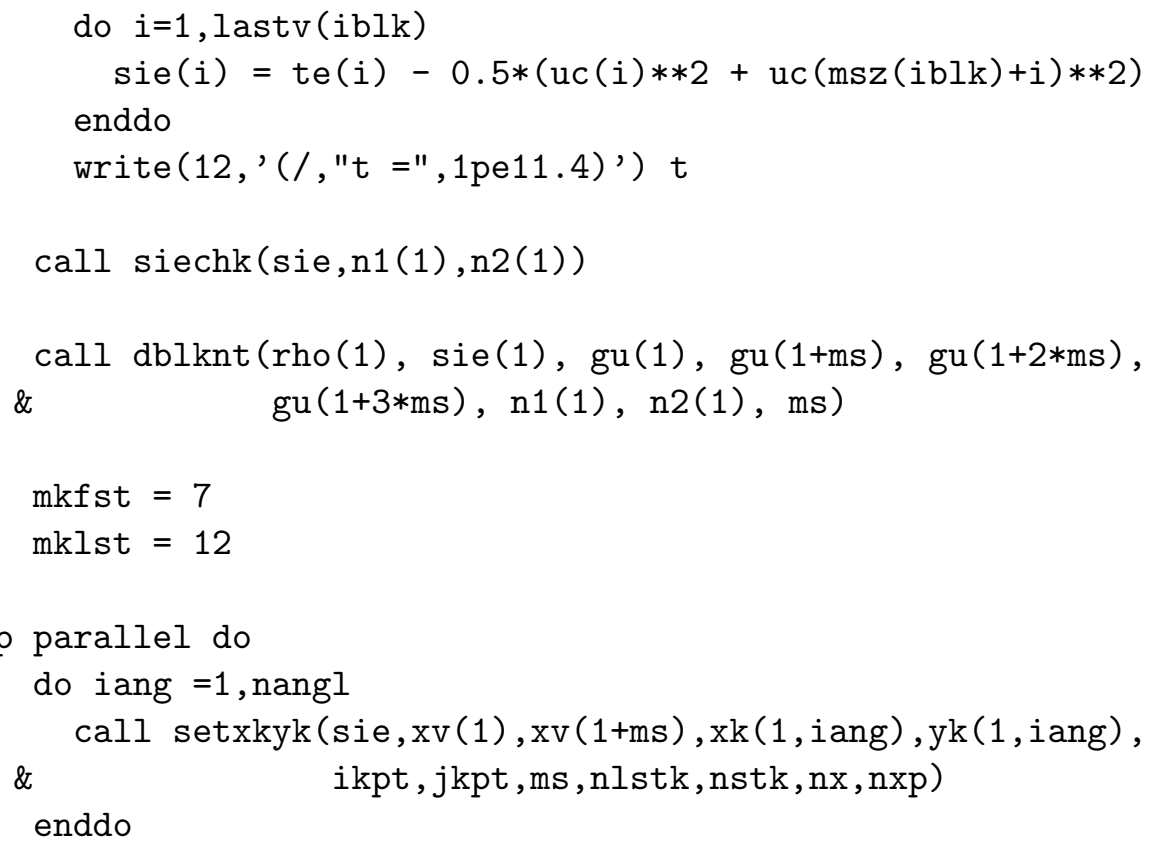

do iang $=1$, nangl

call setxkyk (sie, xv(1), xv(1+ms), xk(1,iang), yk(1,iang),

! \$omp parallel do

!\$omp parallel do private(iang, a01, a03)

! \$omp+ shared (n1,n2, xv, rho, sie,gu, hkint, siband, siirad, hkirad, xk, yk) do 500 iang $=1$, nangl

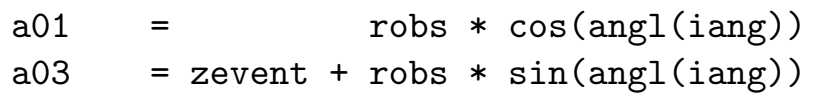

500 continue

do iang=1, nangl

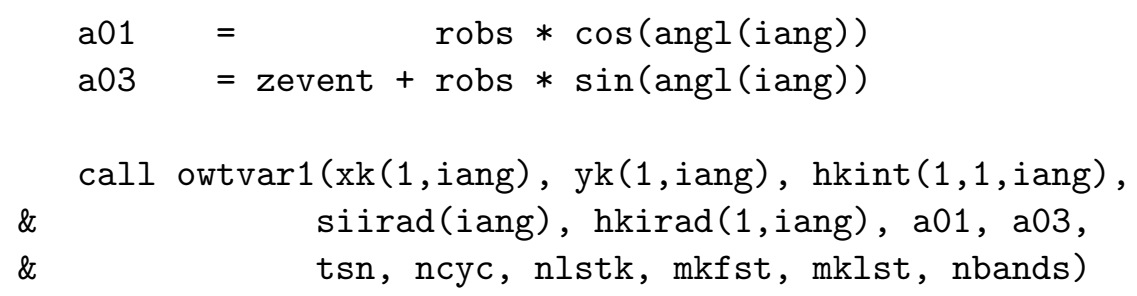

enddo 


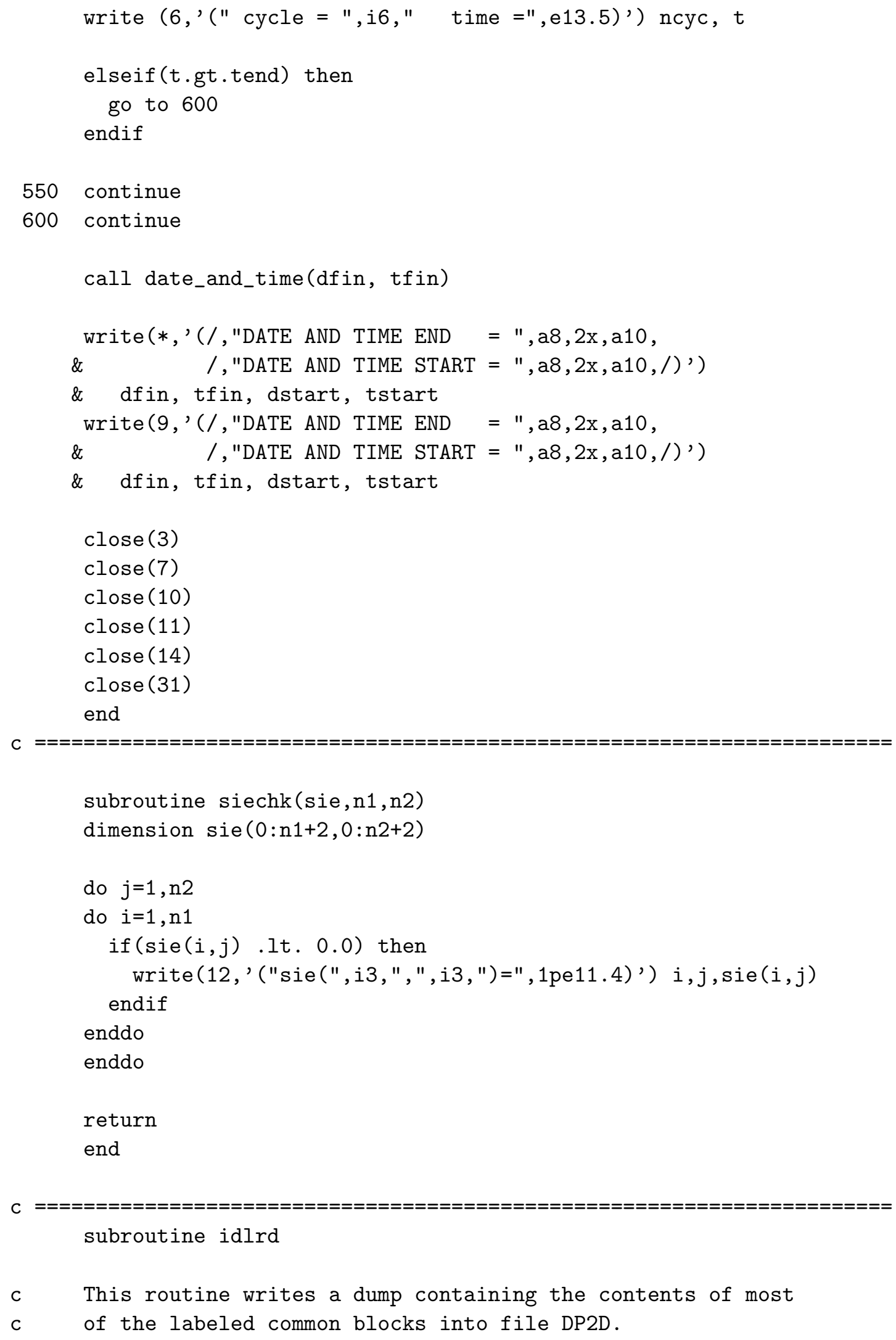




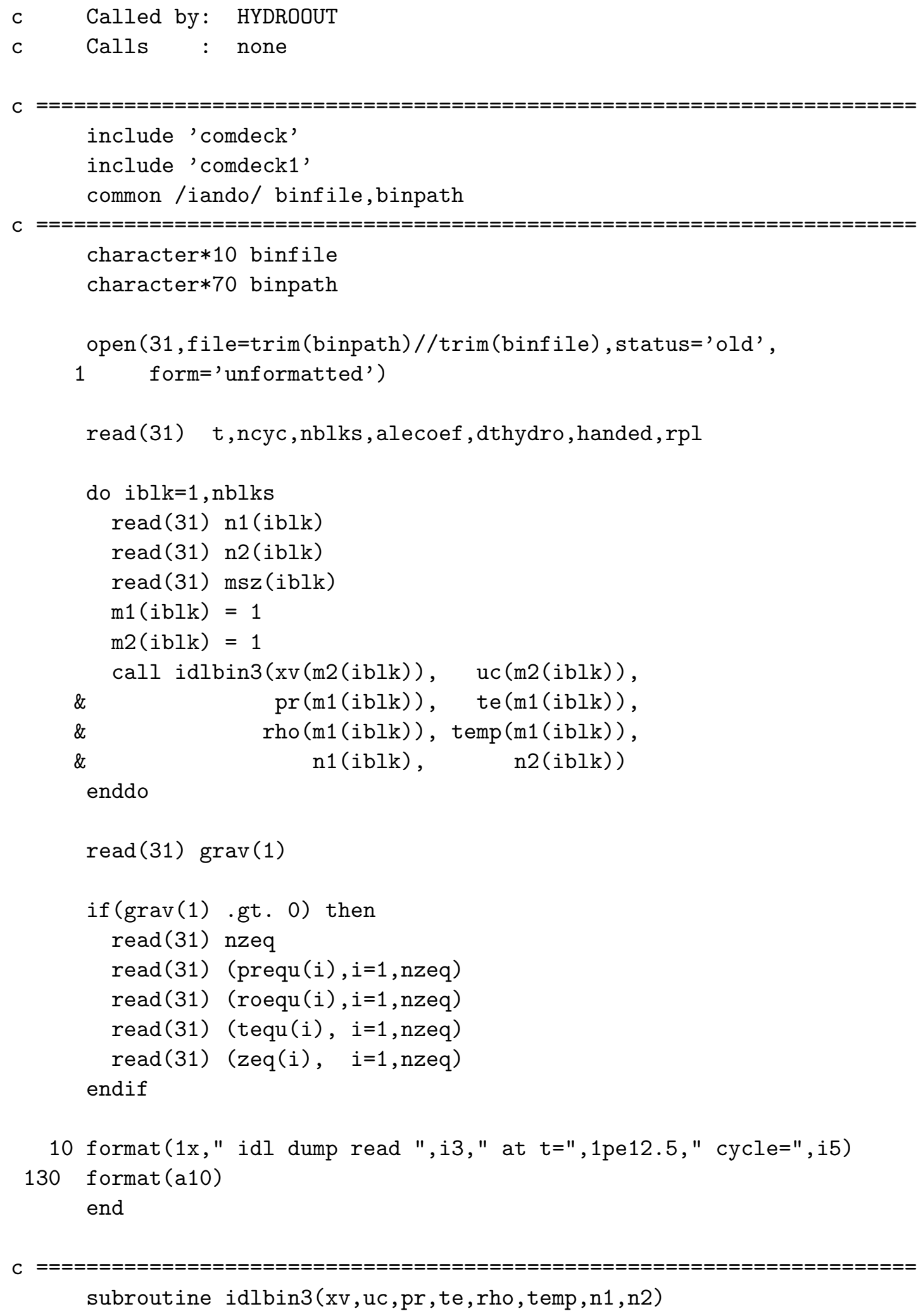




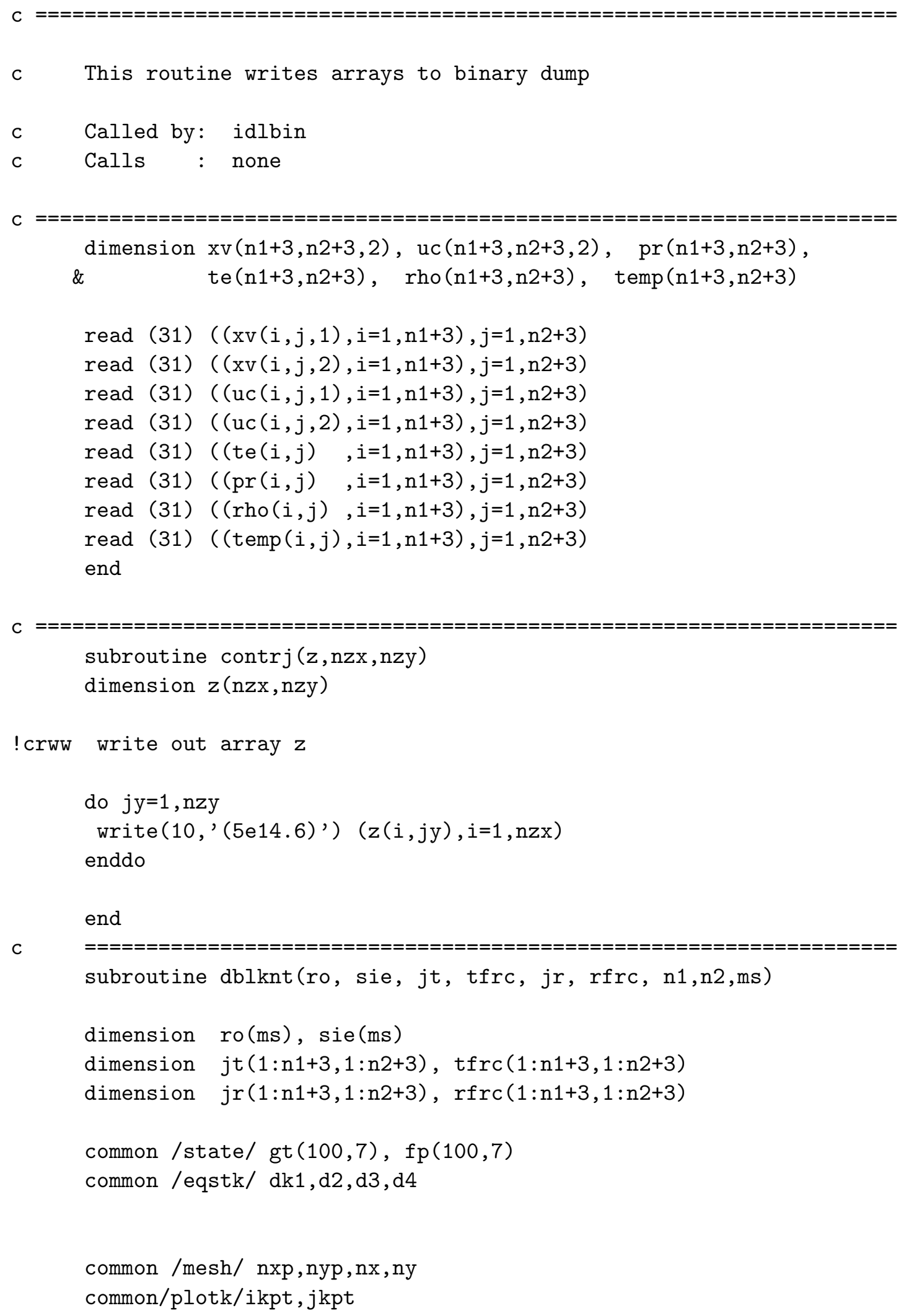




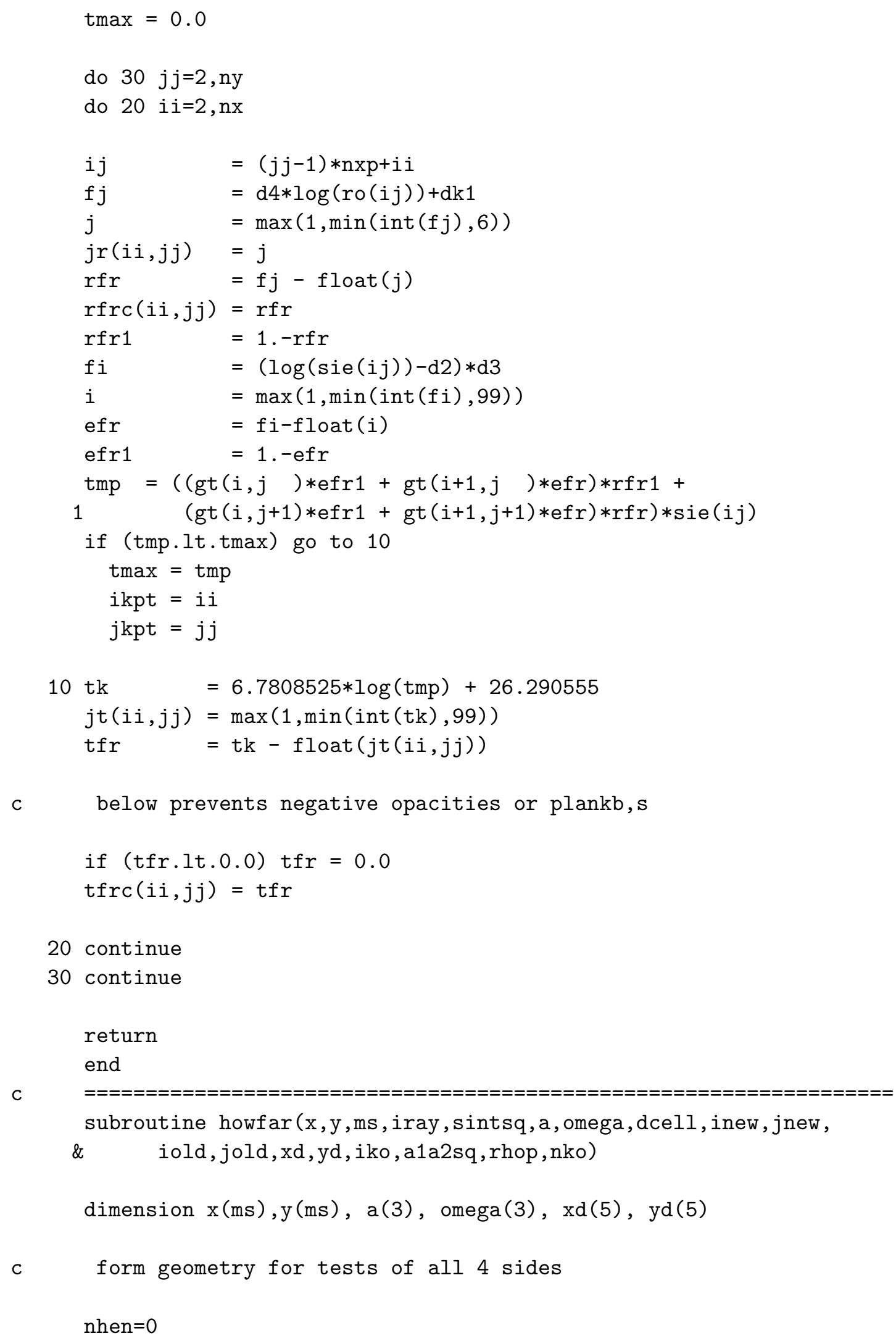




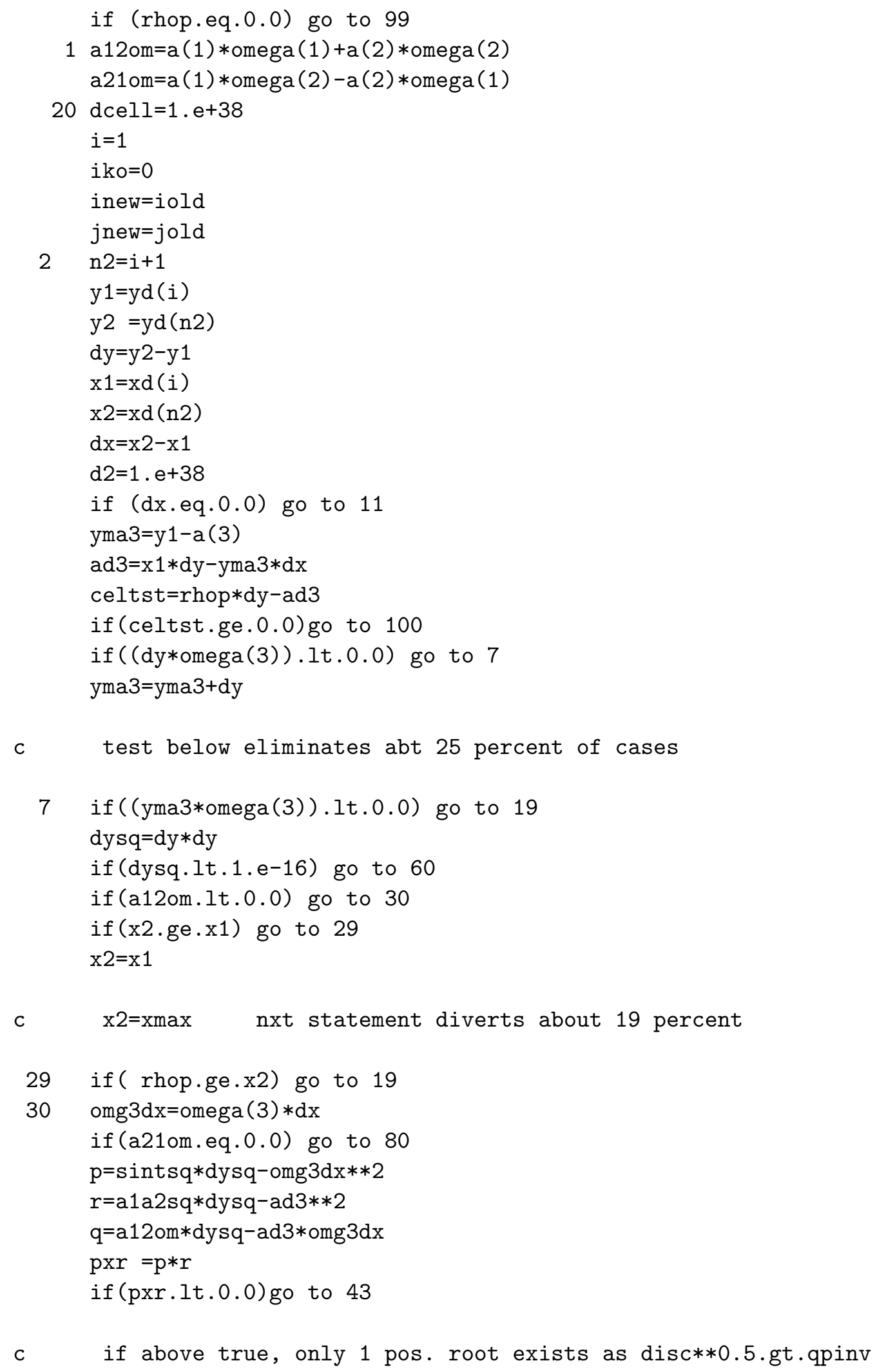




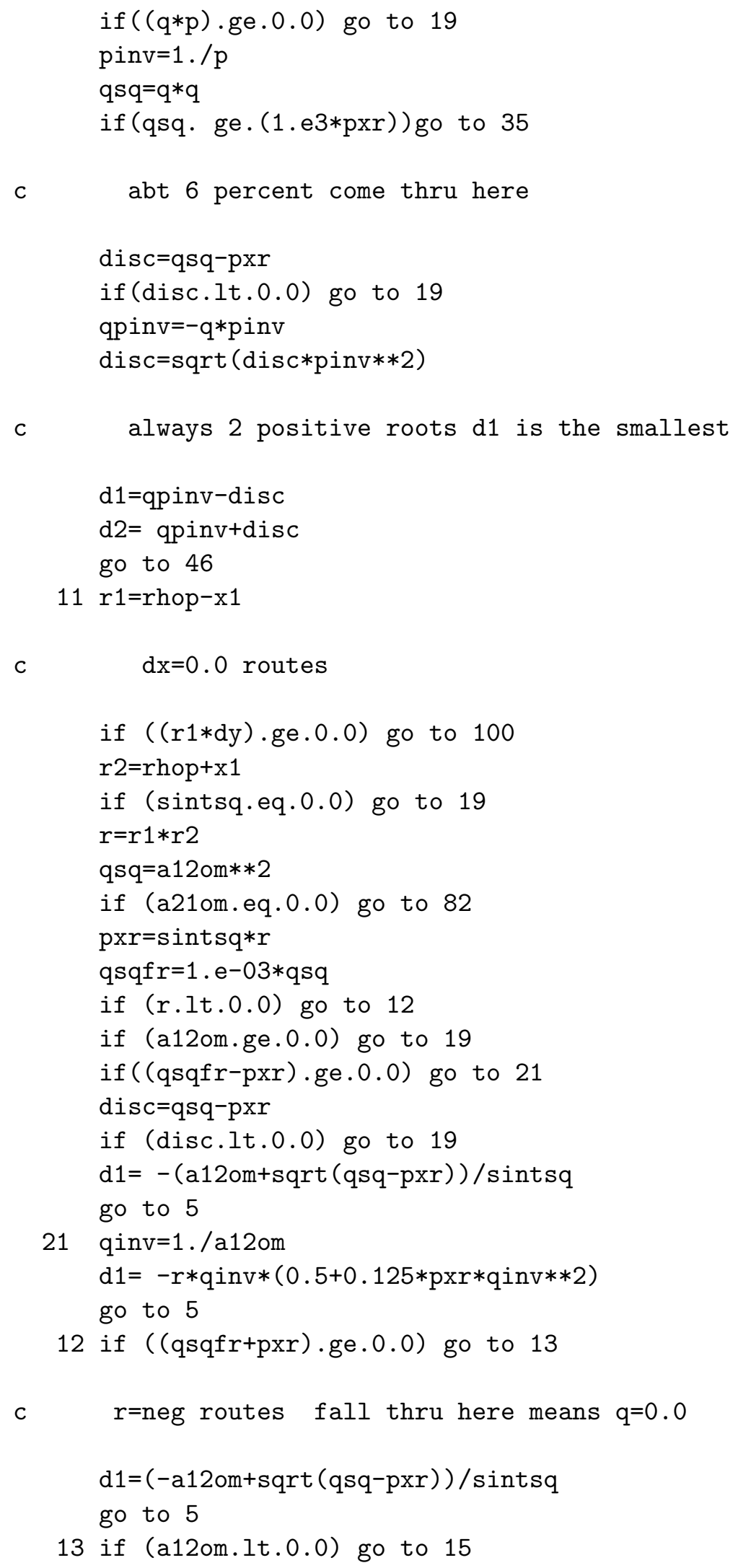




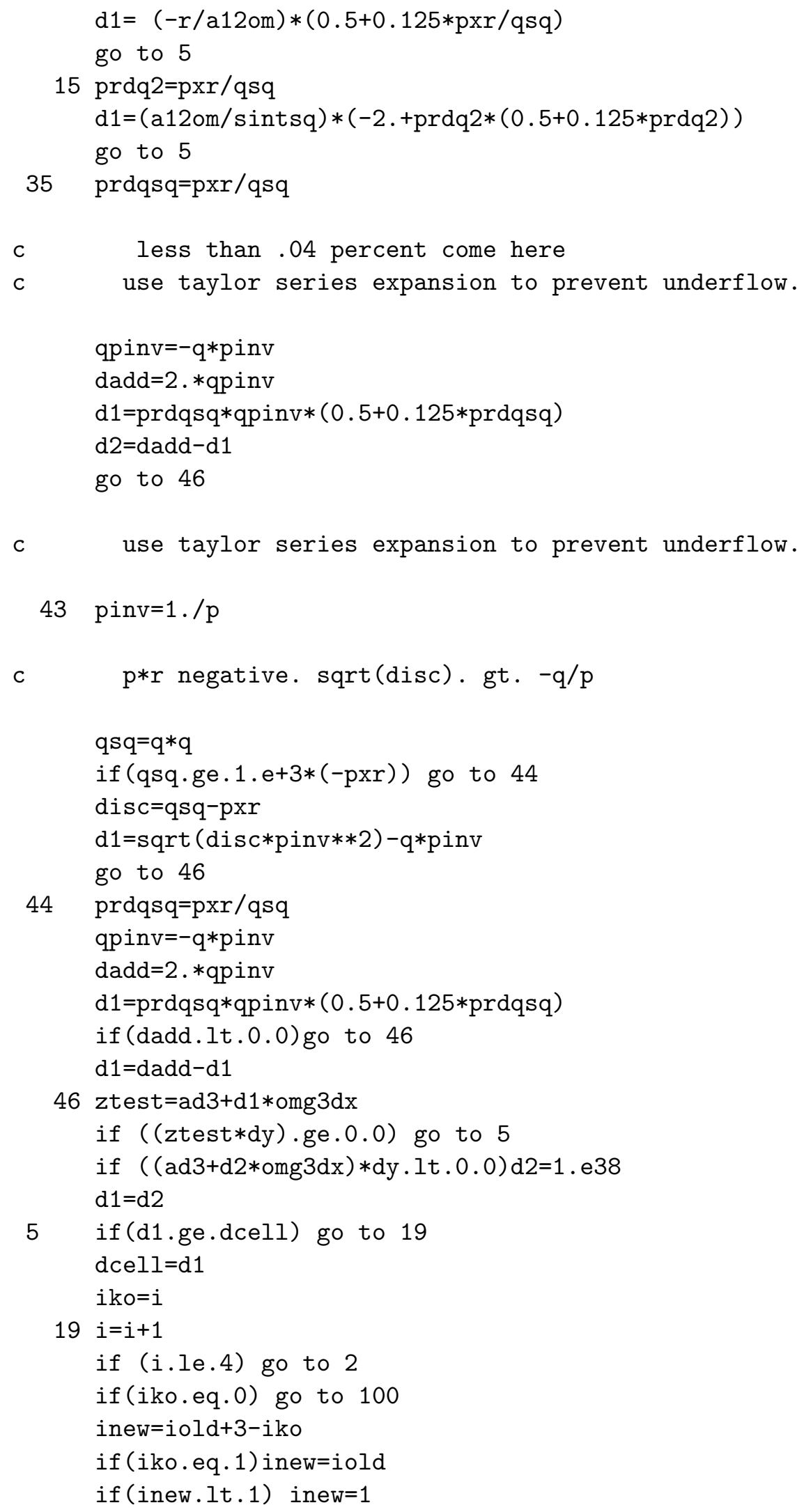




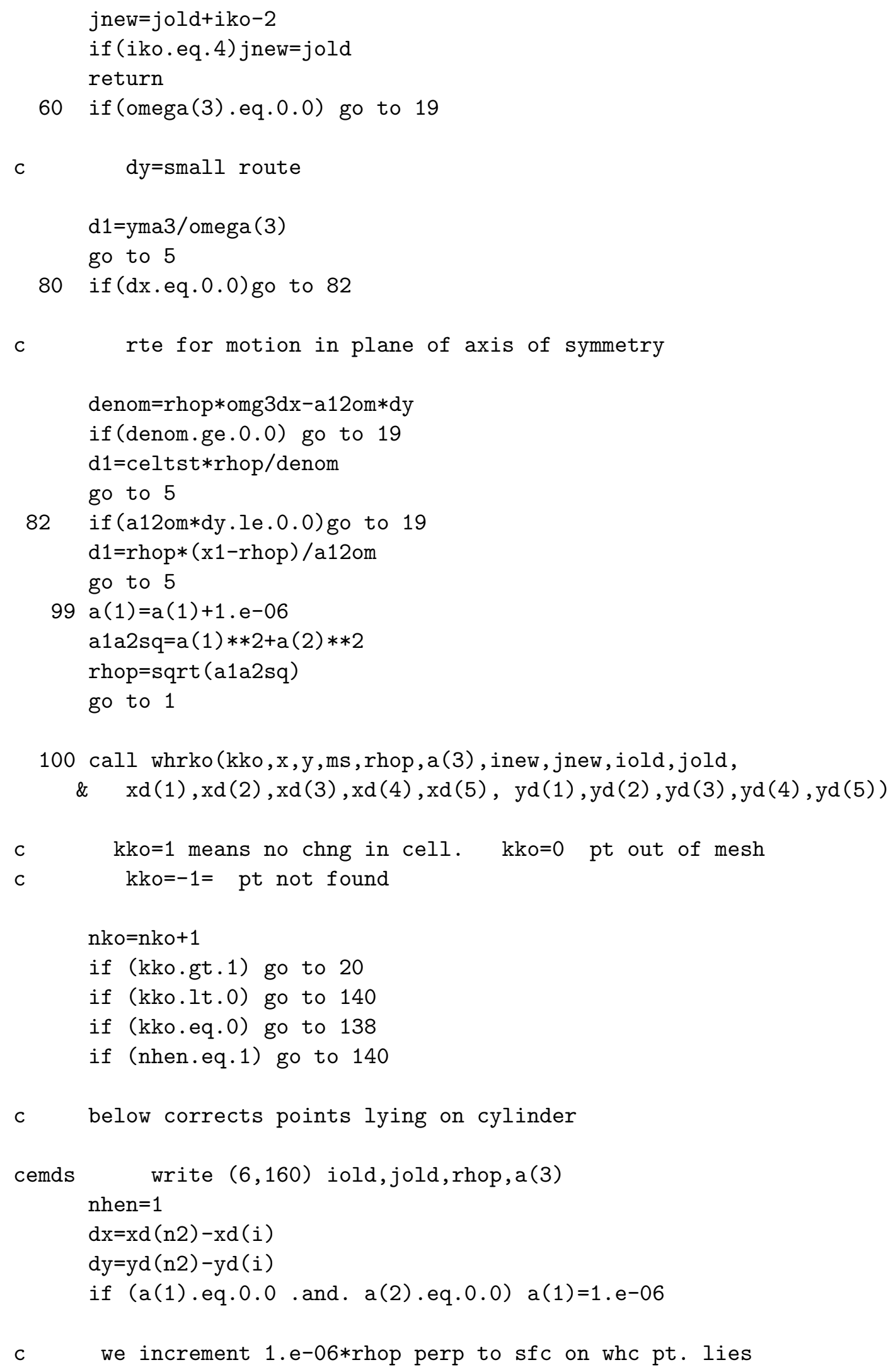




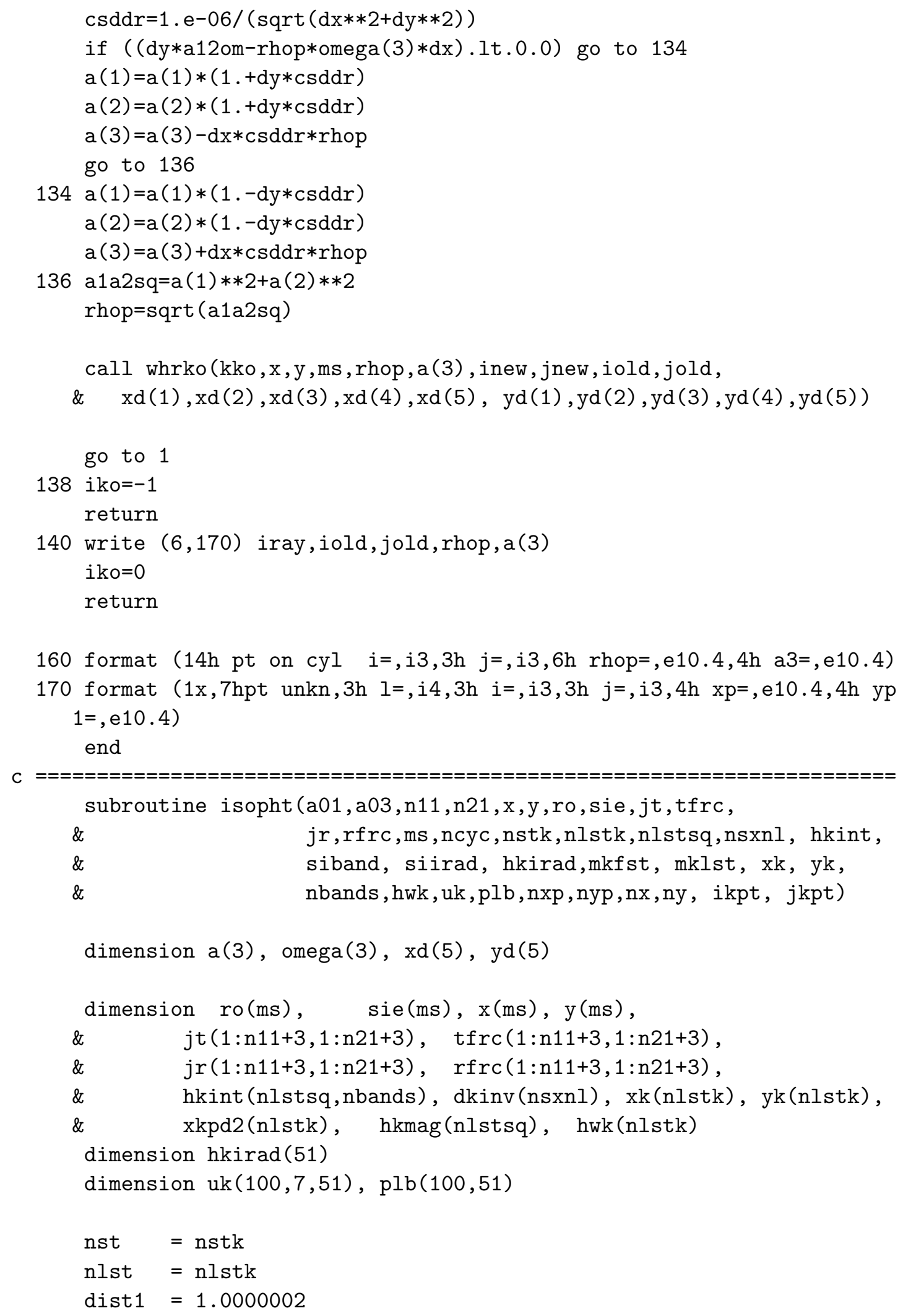




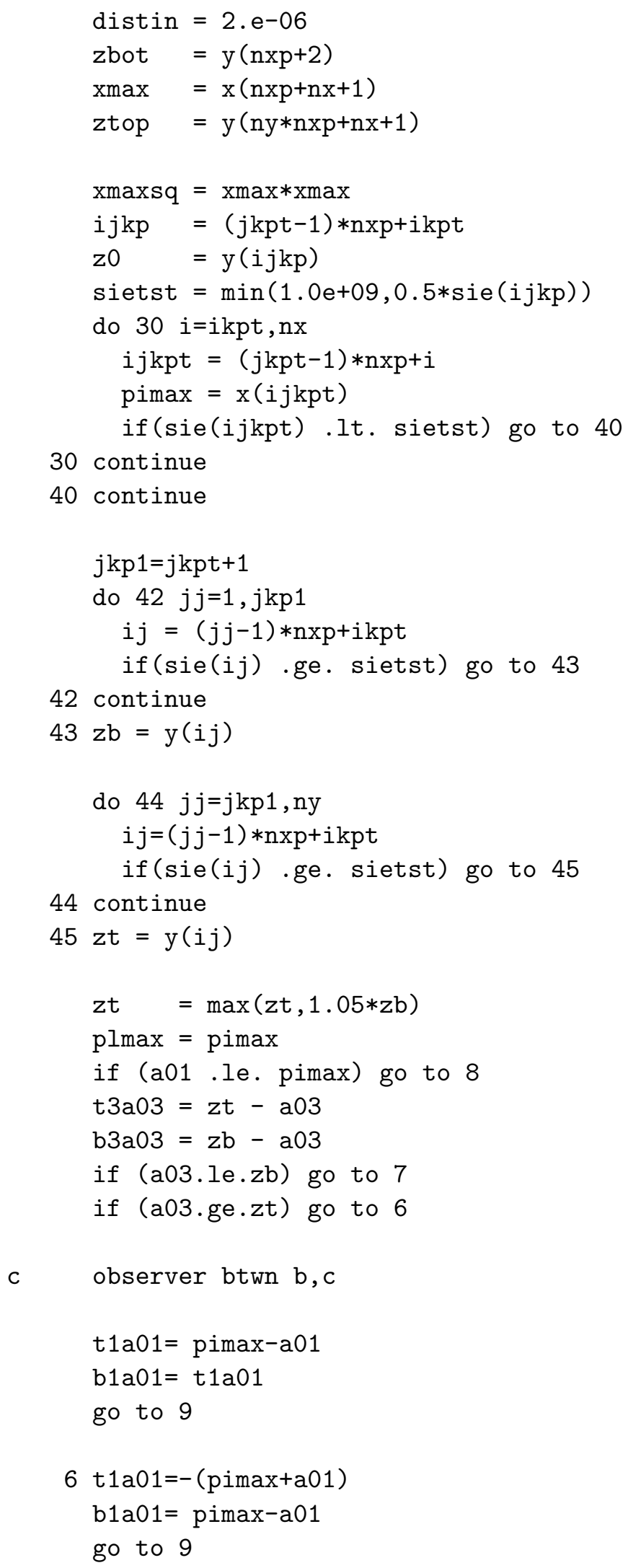




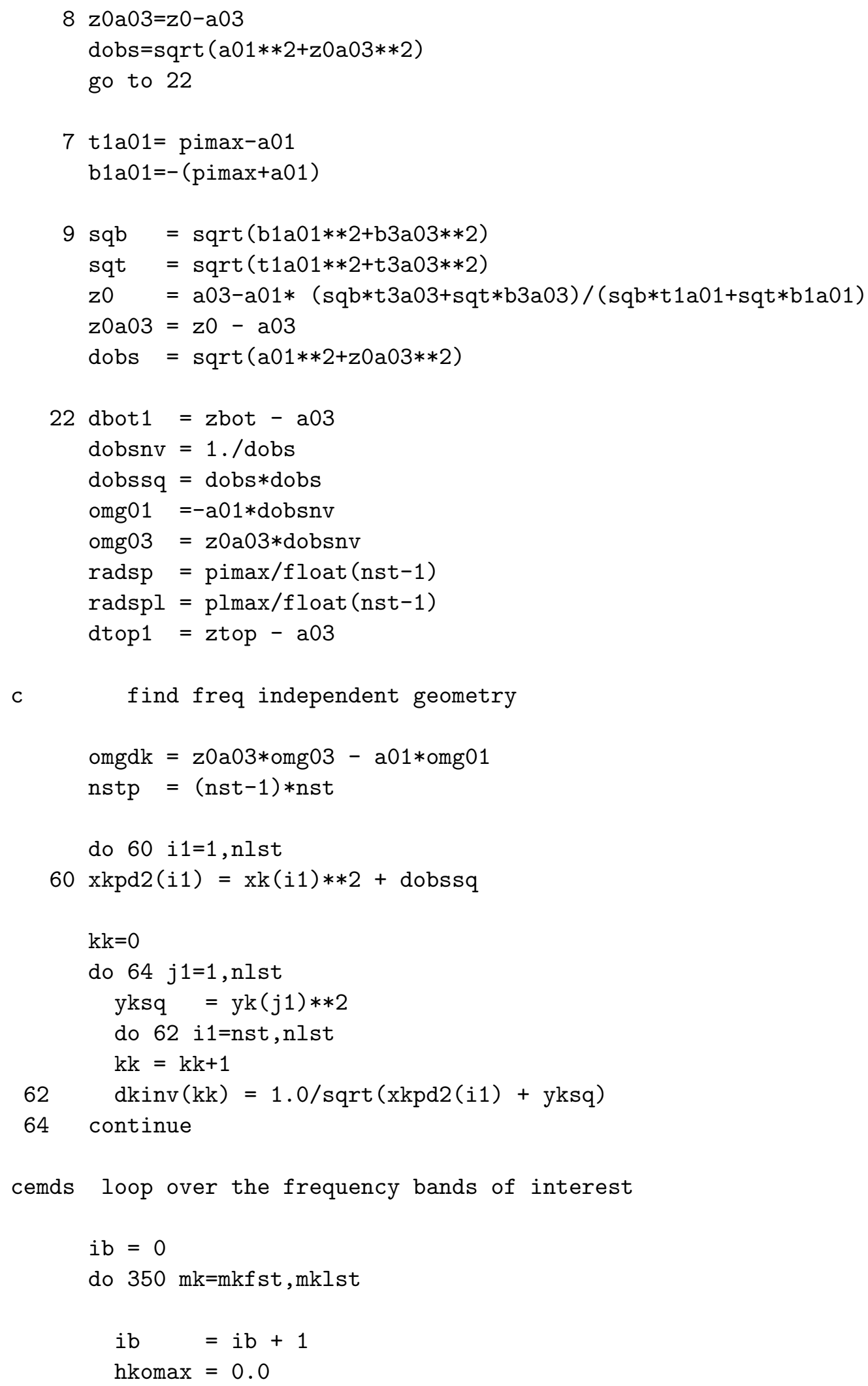




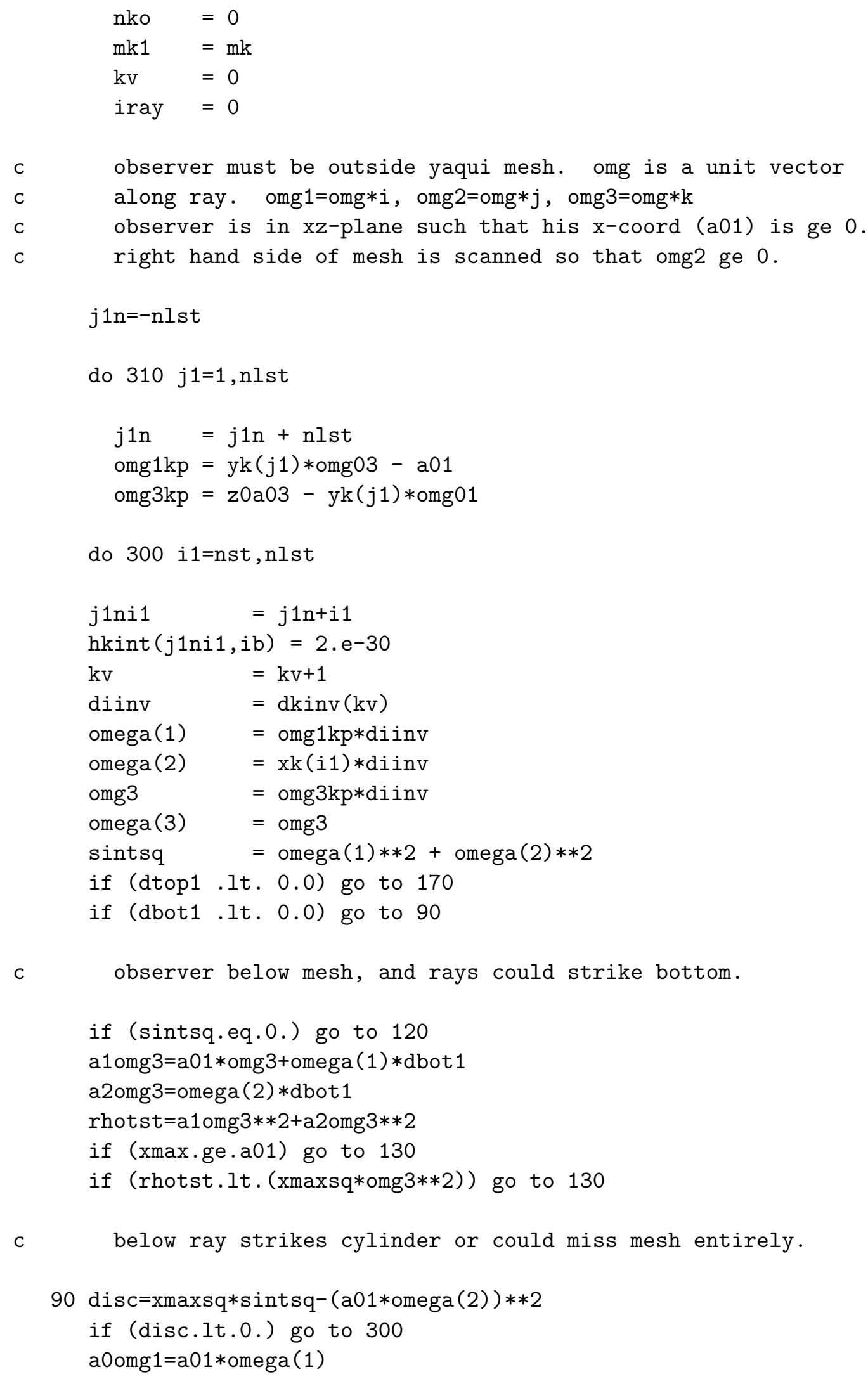




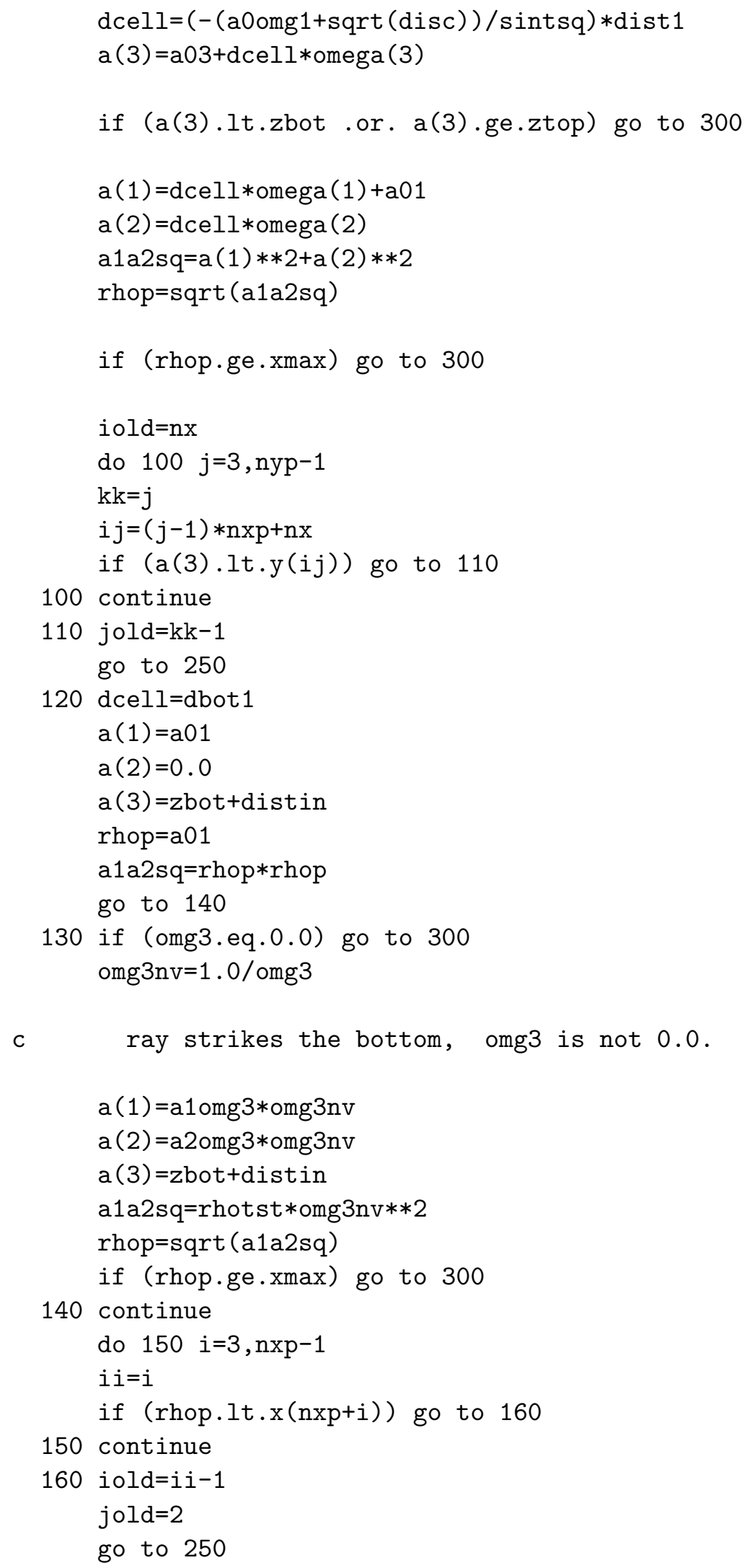




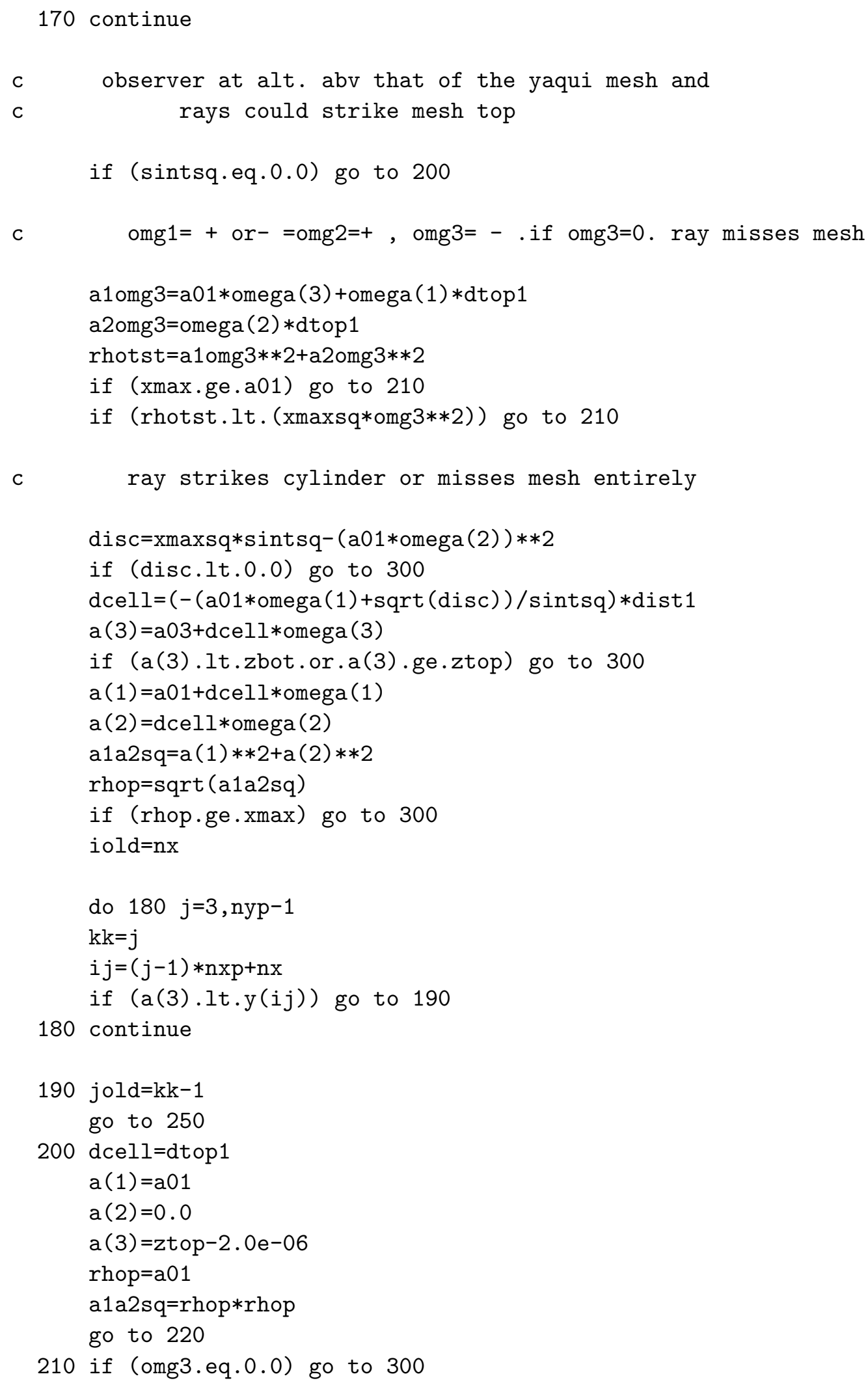




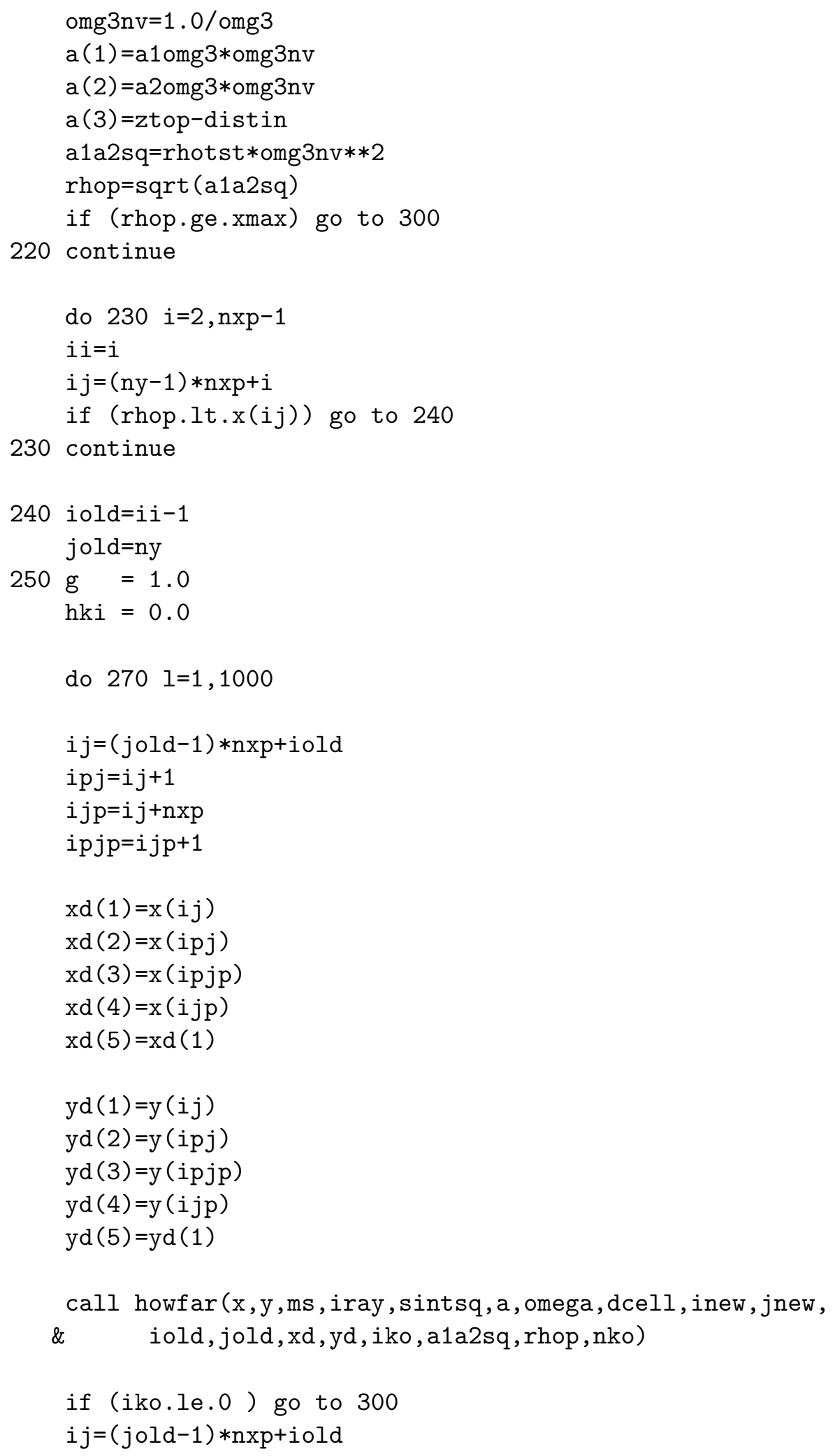




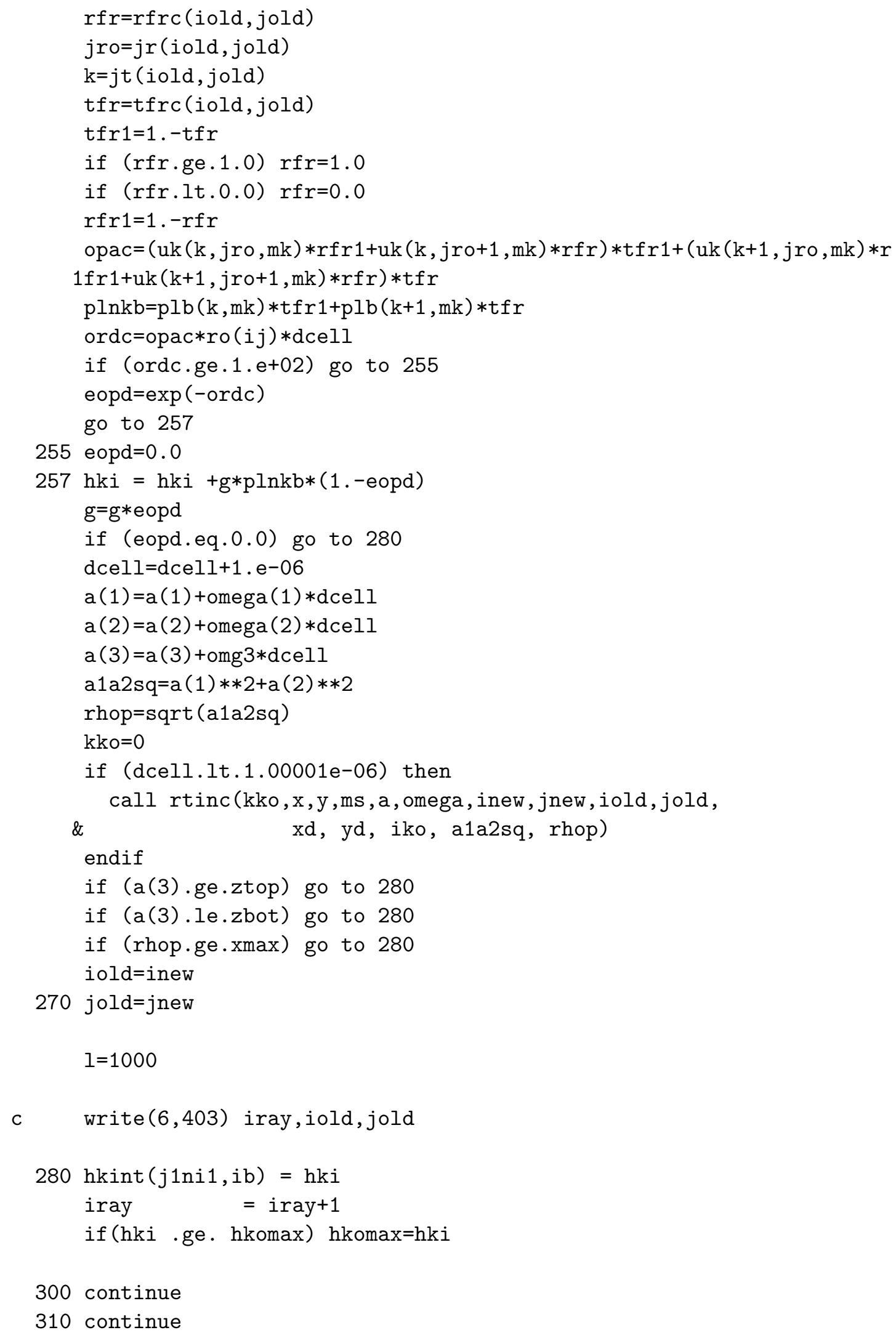


cemds loop over $\mathrm{xk}, \mathrm{yk}$ is now over

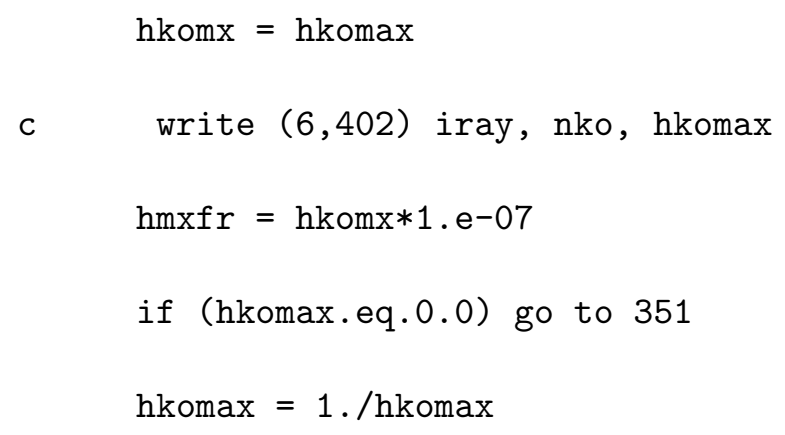


$\mathrm{hkirad}(\mathrm{mk})=2 . * \operatorname{radspl} * \operatorname{radsp} *(\mathrm{~d} 1 \mathrm{irr}+\mathrm{d} 2 \mathrm{irr}) / \mathrm{dobssq}$

350 continue

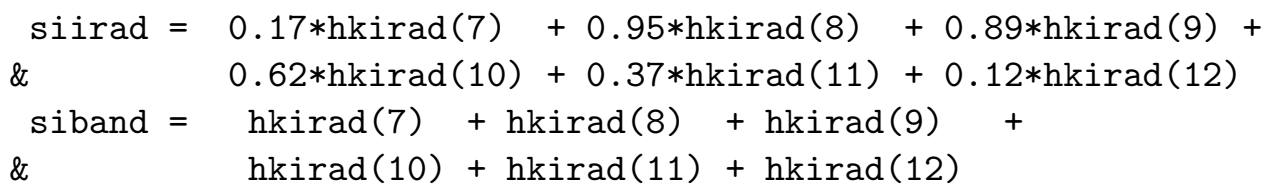

351 continue

return

360 format ( $6 \mathrm{~h}$ a01=,1pe10.3,5h a03=,e10.3,6h dobs=,e10.3,7h omg $1=, e 1$ $10.3,7 \mathrm{~h}$ omg $3=, \mathrm{e} 10.3)$

402 format (9h no rays=,i6,11h wh calls=,i5," hkomax =",1pe11.4)

403 format ( $6 \mathrm{~h}$ iray=,i6, $4 \mathrm{~h} \quad \mathrm{i}=, \mathrm{i} 5,4 \mathrm{~h} \quad \mathrm{j}=, \mathrm{i} 4$ )

700 format $(11(1 \mathrm{x}$, e9.3))

702 format(12h intensities)

end

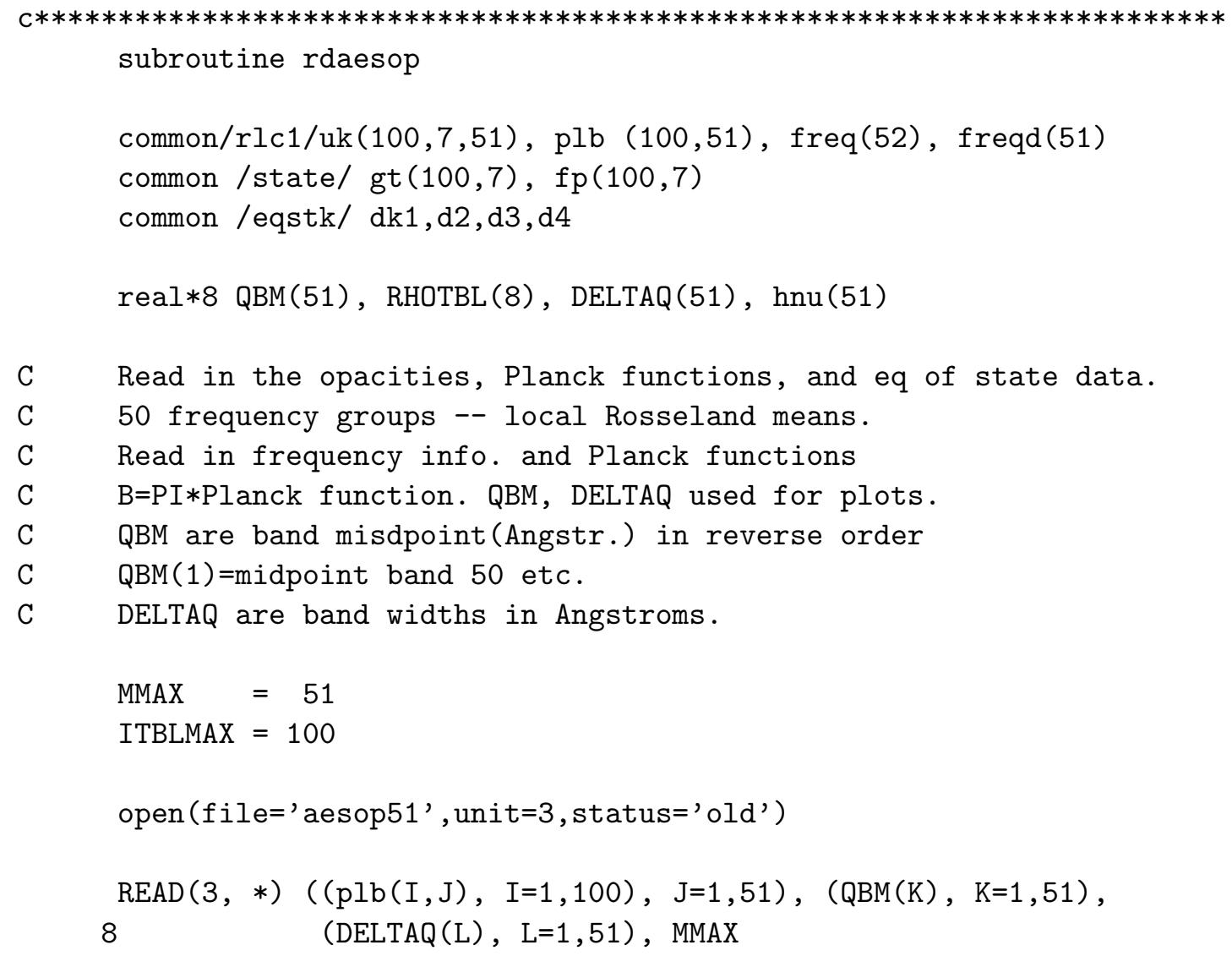




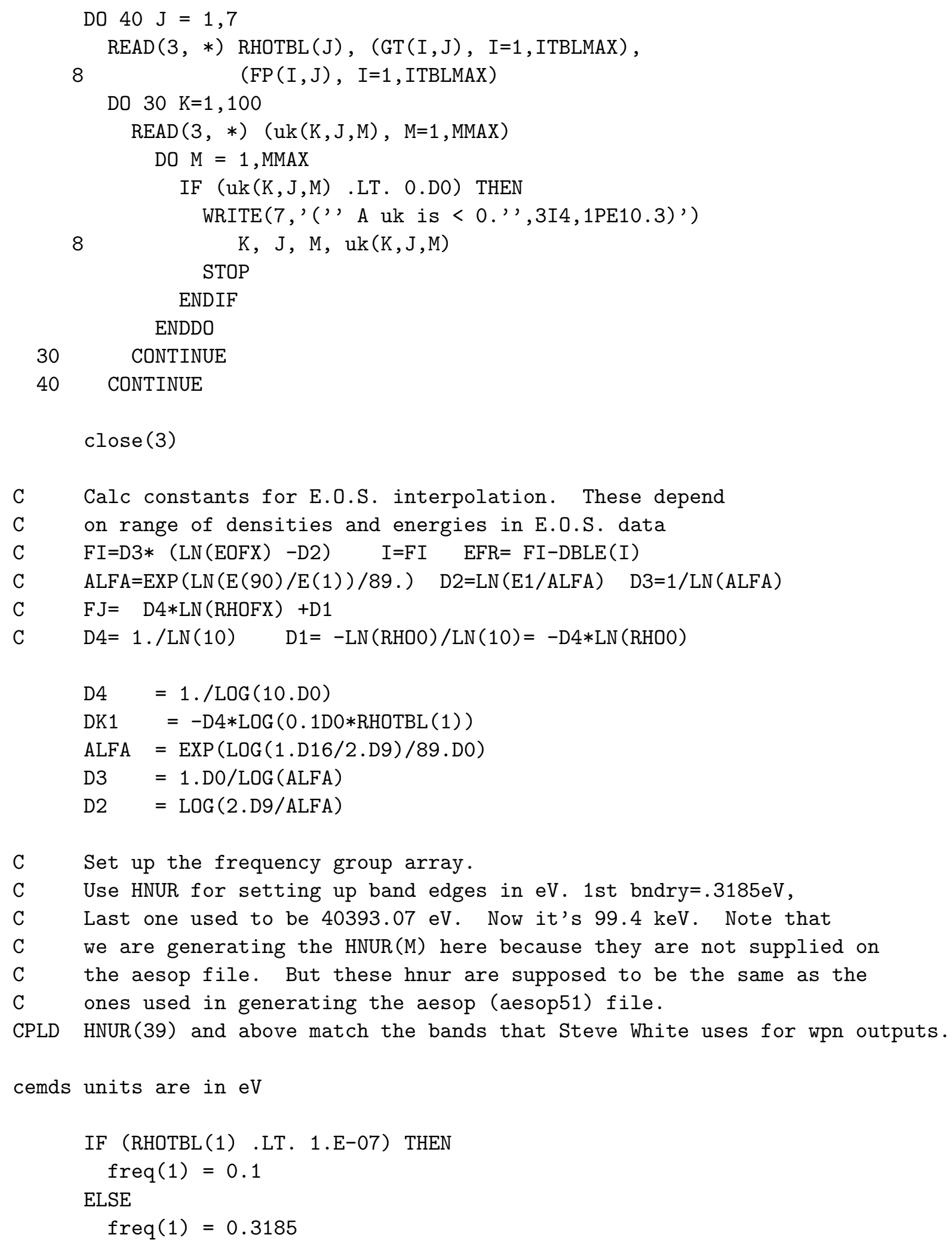




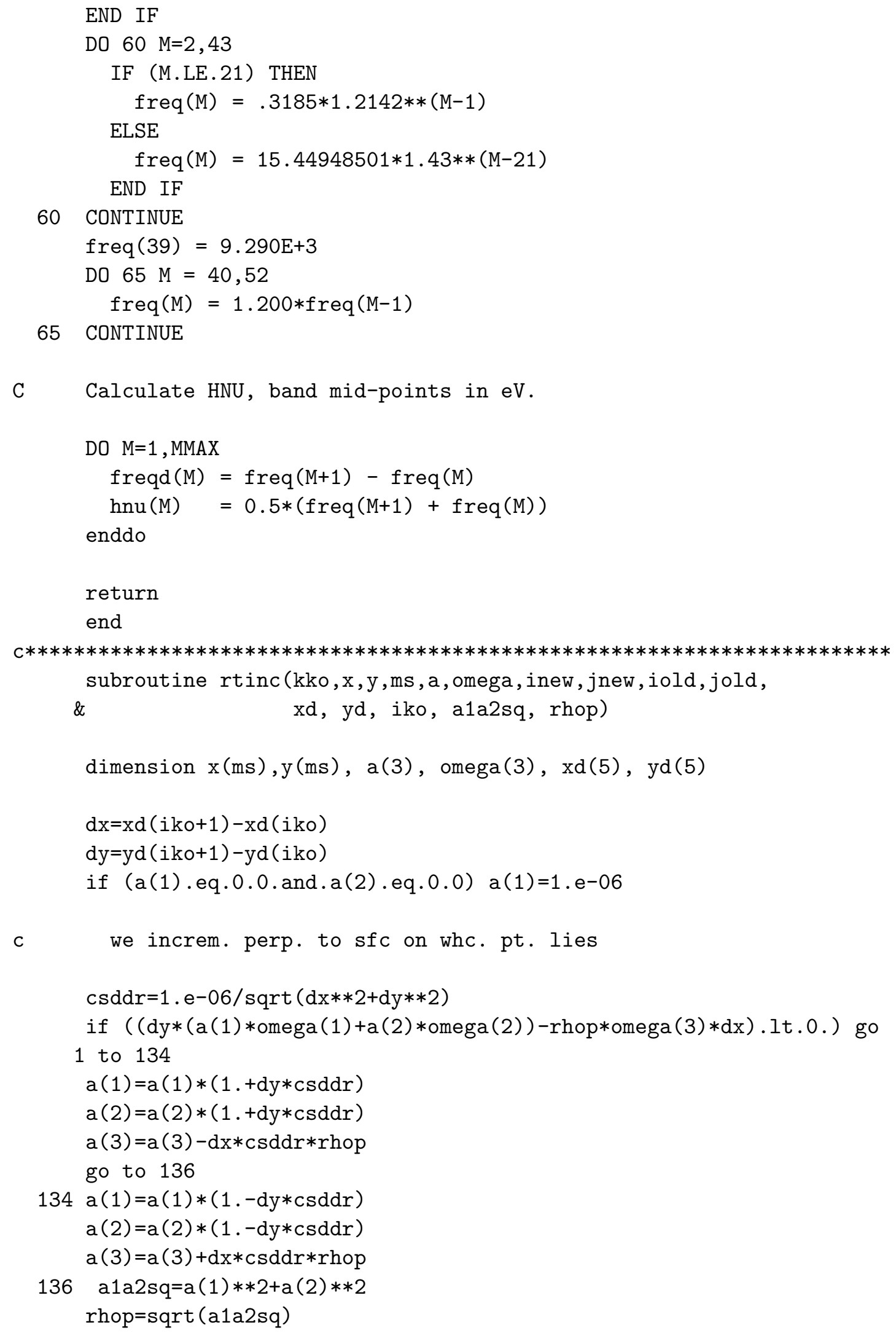


call whrko(kko, $x, y, m s$, rhop, a (3), inew, jnew, iold, jold,

\& $\quad x d(1), x d(2), x d(3), x d(4), x d(5), y d(1), y d(2), y d(3), y d(4), y d(5))$

return

end

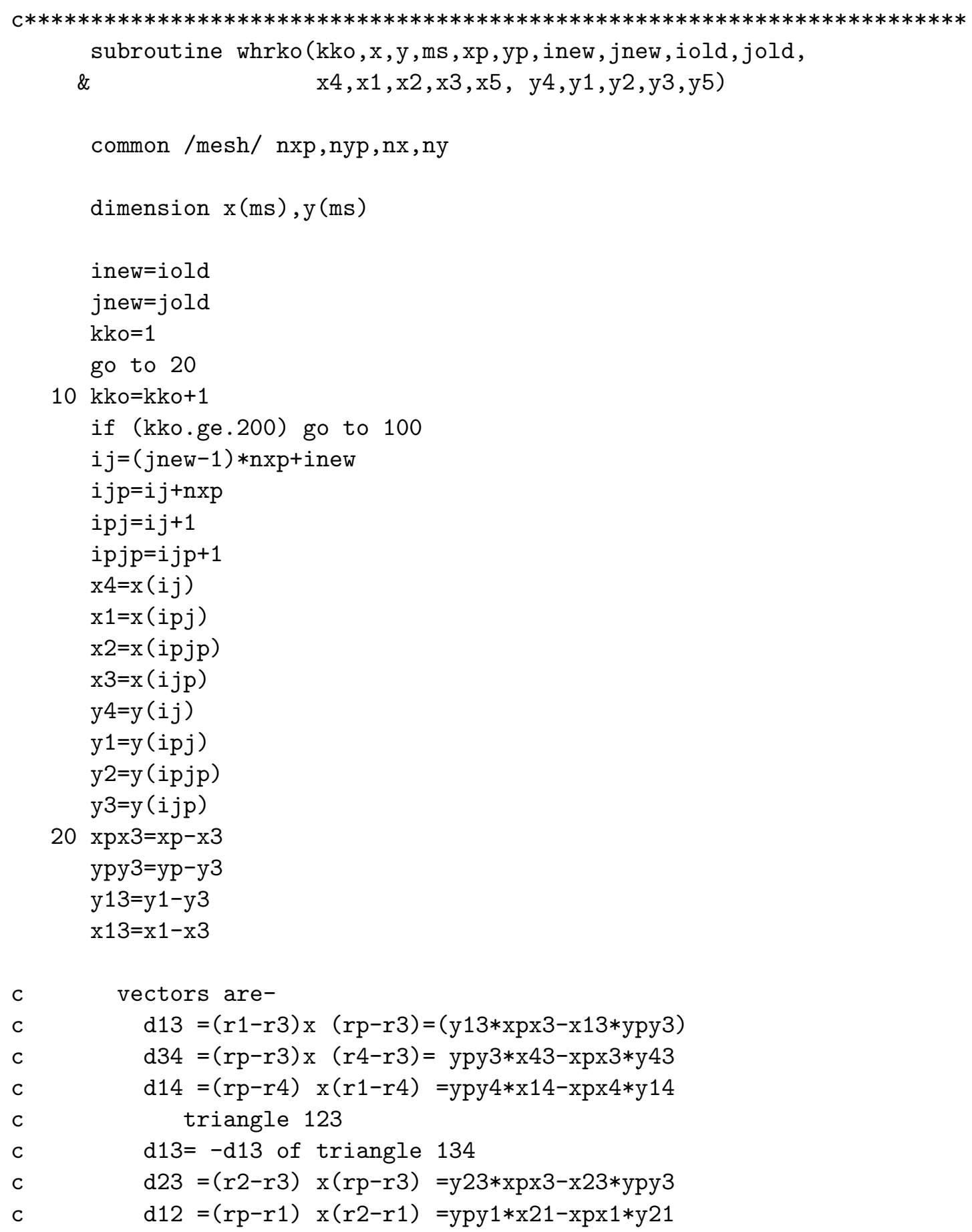




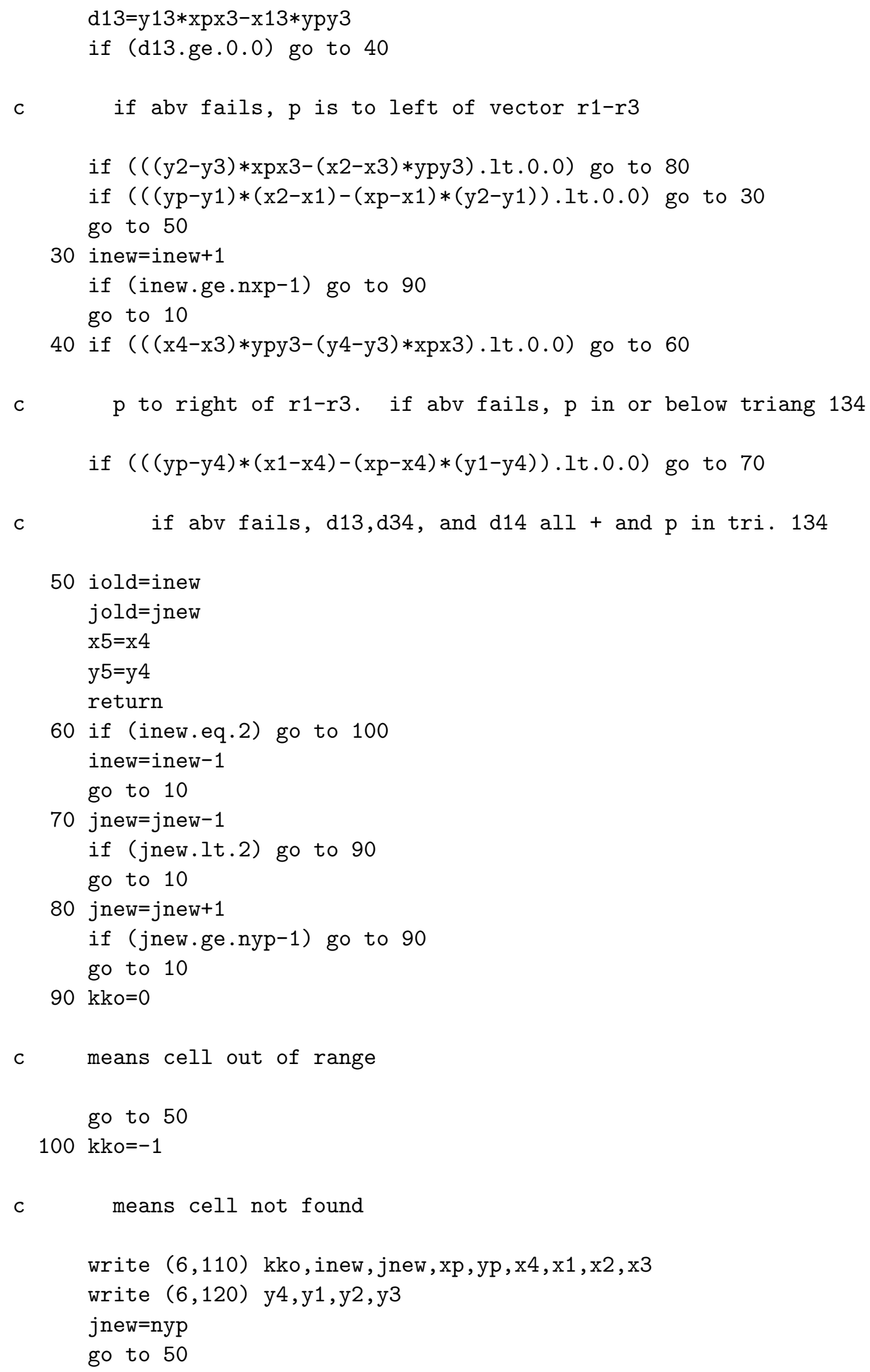




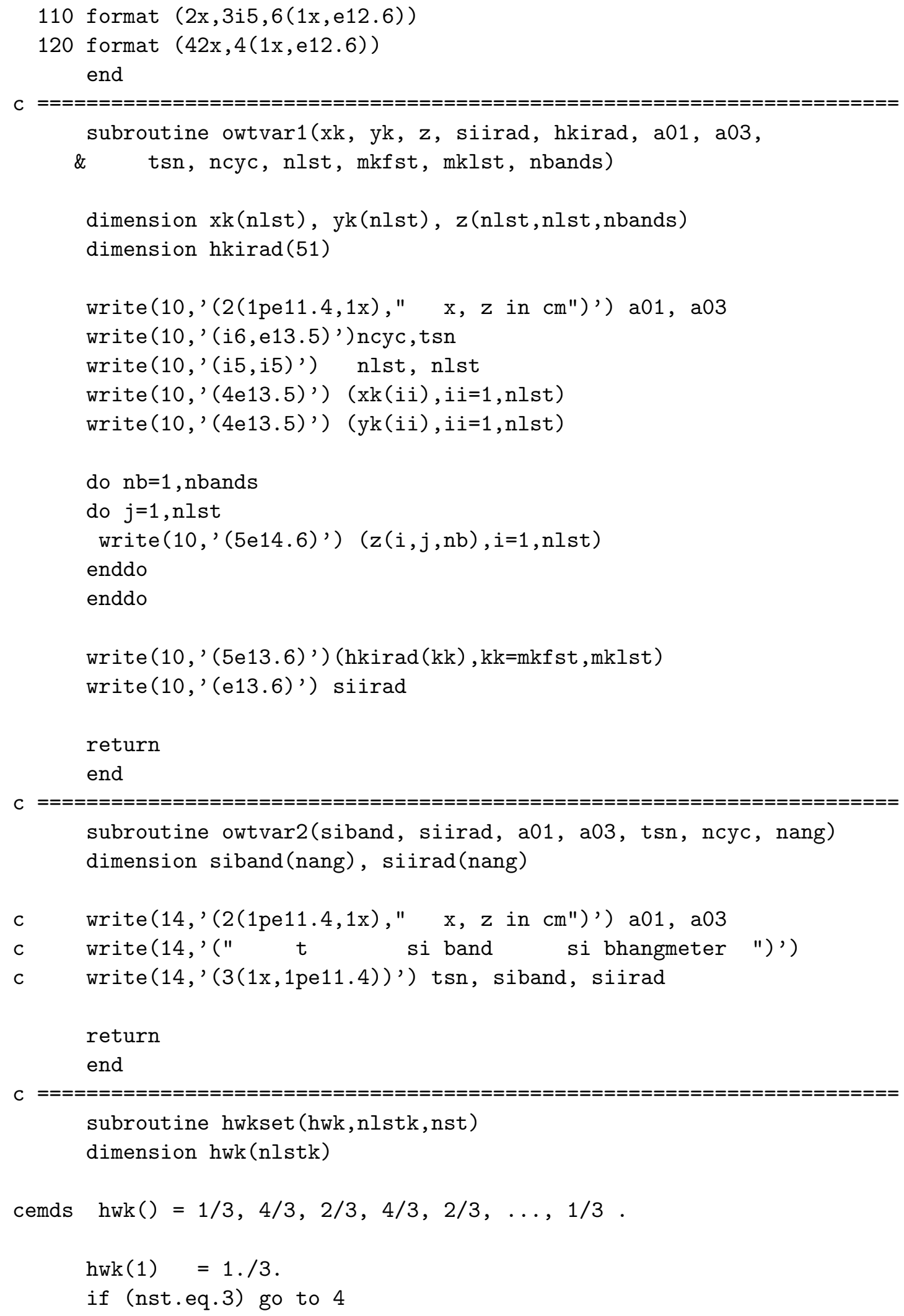




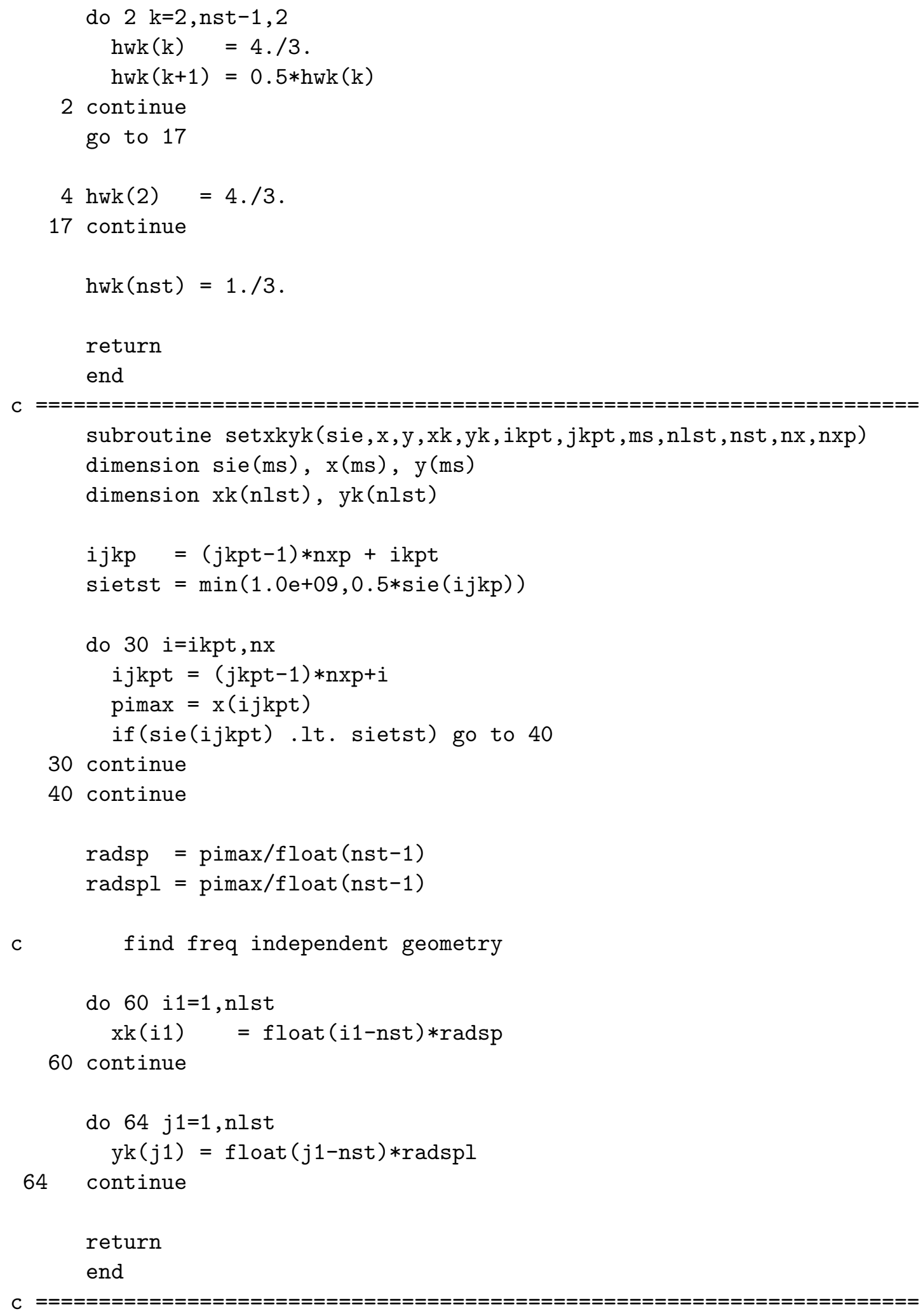




\subsection{OpenMP make file on a MAC G5}

Next we give a listing of the make file used on a four processor Mac.

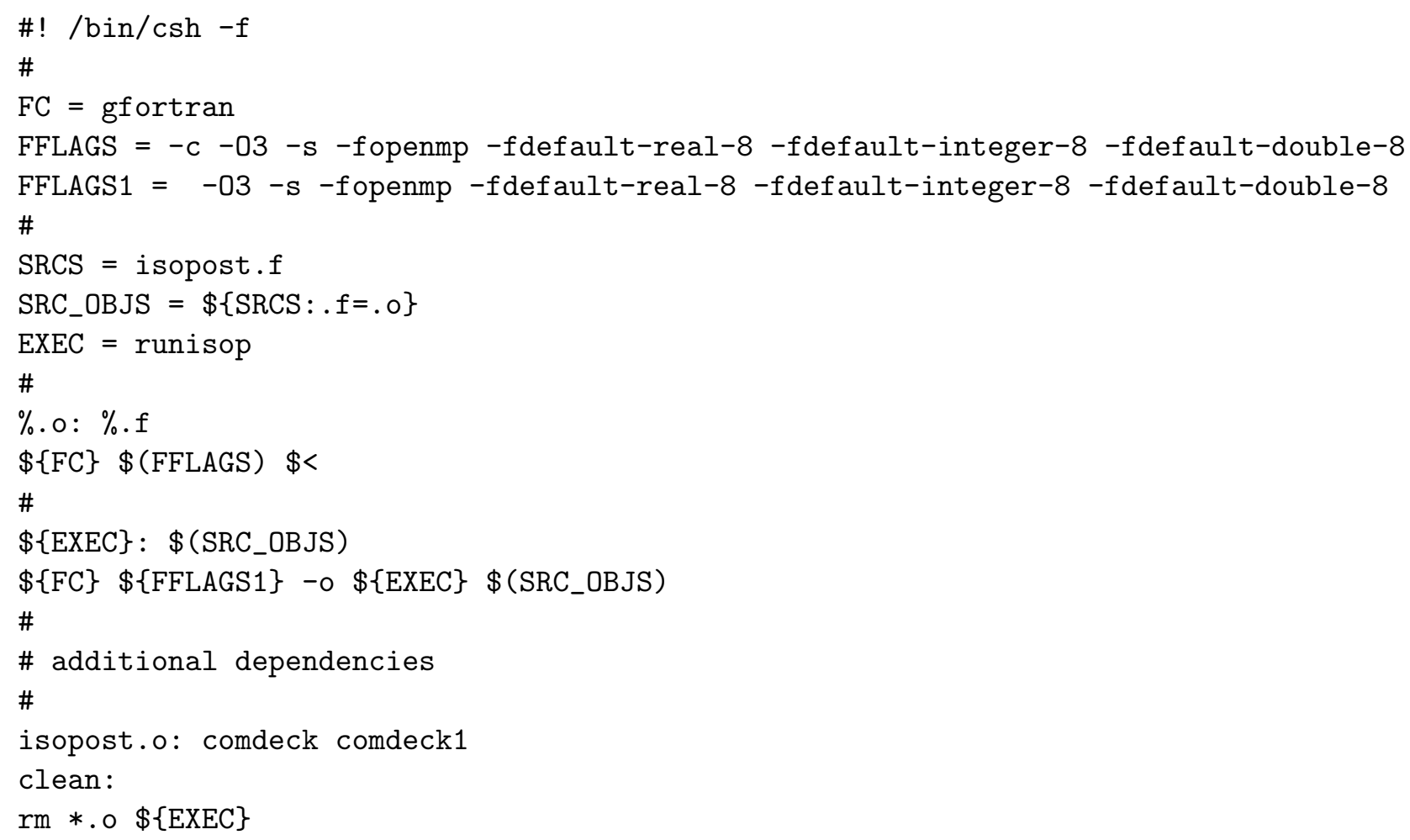

\subsection{Make file for serial processor such as Sun}

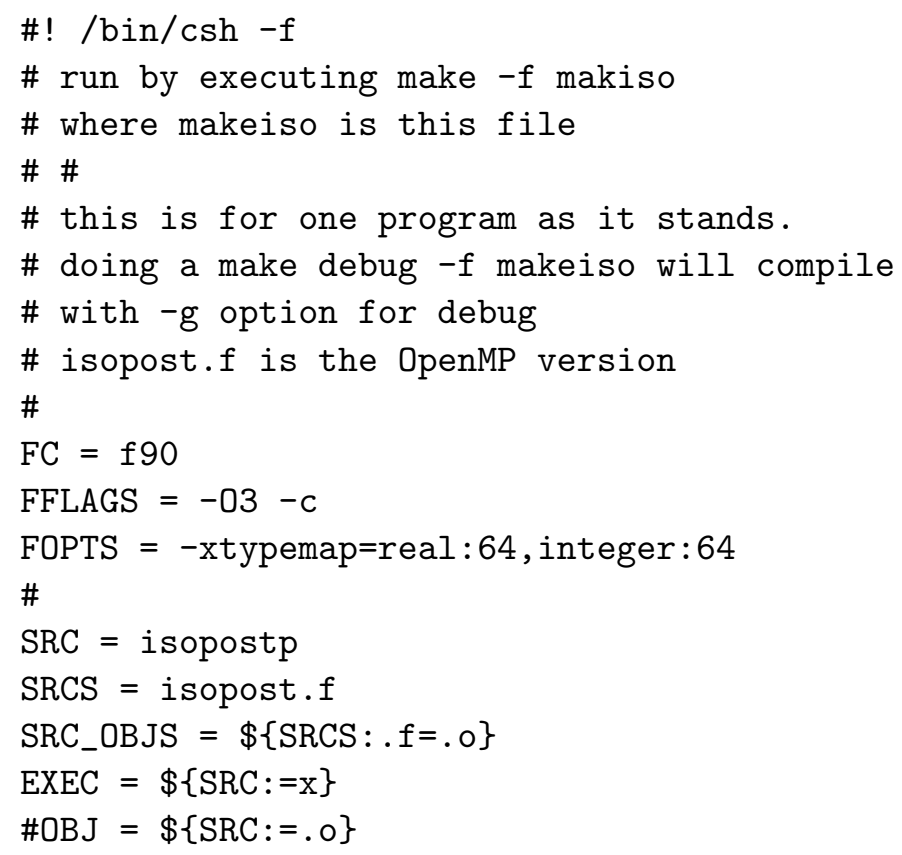




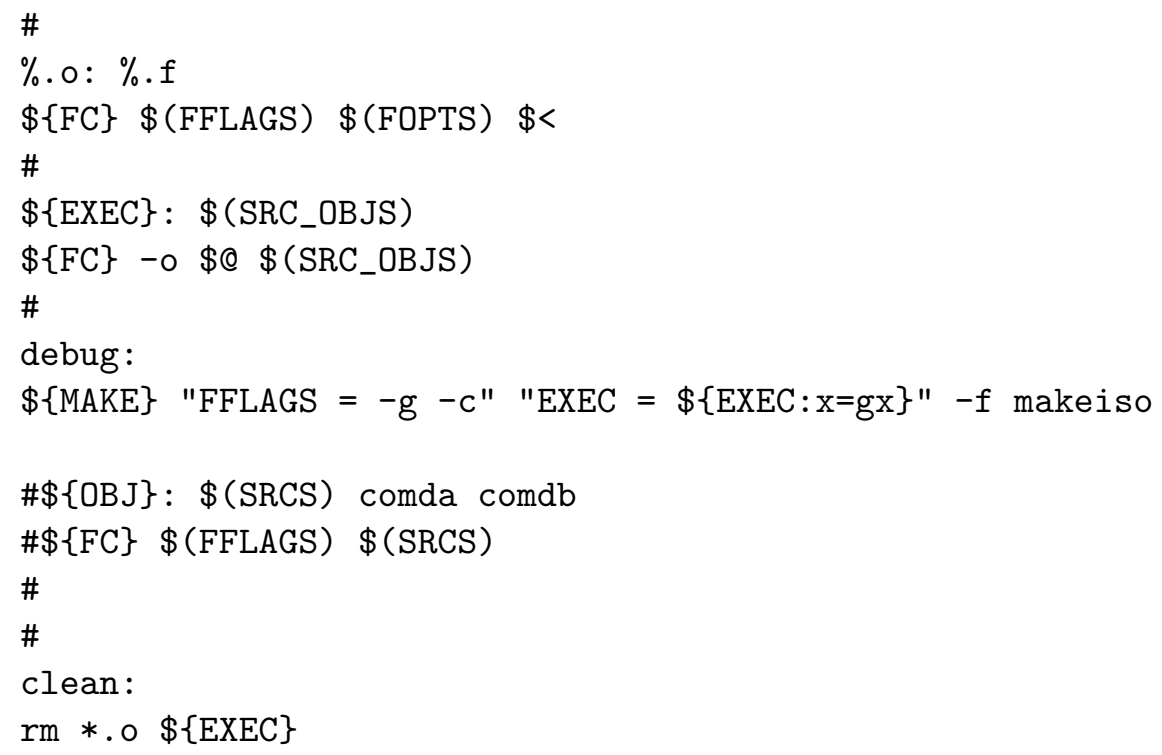


This report has been reproduced directly from the best available copy. It is available electronically on the Web (http://www.doe.gov/bridge).

Copies are available for sale to U.S. Department of Energy employees and contractors from:

Office of Scientific and Technical Information

P.O. Box 62

Oak Ridge, TN 37831

(865) 576-8401

Copies are available for sale to the public from: National Technical Information Service

U.S. Department of Commerce

5285 Port Royal Road

Springfield, VA 22161

(800) 553-6847 
- Los Alamos NATIONAL LABORATORY

EST.1943 